\title{
Build systems à la carte: Theory and practice
}

\author{
ANDREY MOKHOV(iD \\ School of Engineering, Newcastle University, Newcastle upon Tyne, UK \\ Jane Street, London, UK \\ (e-mail: andrey.mokhov@ncl.ac.uk) \\ NEIL MITCHELL \\ Facebook, London, UK \\ (e-mail: ndmitchell@gmail.com)
}

SIMON PEYTON JONES

Microsoft Research, Cambridge, UK

(e-mail: simonpj@microsoft.com)

\begin{abstract}
Build systems are awesome, terrifying - and unloved. They are used by every developer around the world, but are rarely the object of study. In this paper, we offer a systematic, and executable, framework for developing and comparing build systems, viewing them as related points in a landscape rather than as isolated phenomena. By teasing apart existing build systems, we can recombine their components, allowing us to prototype new build systems with desired properties.
\end{abstract}

\section{Introduction}

Build systems (such as MAKE) are big, complicated, and used by every software developer on the planet. But they are a sadly unloved part of the software ecosystem, very much a means to an end, and seldom the focus of attention. For years MAKE dominated, but more recently the challenges of scale have driven large software firms like Microsoft, Facebook, and Google to develop their own build systems, exploring new points in the design space. These complex build systems use subtle algorithms, but they are often hidden away, and not the object of study.

In this paper, we give a general framework in which to understand and compare build systems, in a way that is both abstract (omitting incidental detail) and yet precise (implemented as Haskell code). Specifically, we make these contributions:

- Build systems vary on many axes, including: static versus dynamic dependencies; local versus cloud; deterministic versus non-deterministic build tasks; early cutoff; self-tracking build systems; and the type of persistently stored build information. In Section 2, we identify some of these key properties, illustrated by four carefully chosen build systems. 
- We describe some simple but novel abstractions that crisply encapsulate what a build system is (Section 3), allowing us, for example, to speak about what it means for a build system to be correct.

- We identify two key design choices that are typically deeply wired into any build system: the order in which tasks are built (Section 4) and whether or not a task is rebuilt (Section 5). These choices turn out to be orthogonal, which leads us to a new classification of the design space (Section 6).

- We show that we can instantiate our abstractions to describe the essence of a variety of different real-life build systems, including MAKE, SHAKE, BAZEL, BUCK, NiX, and EXCEL, ${ }^{1}$ each by the composition of the two design choices (Section 6). Doing this modelling in a single setting allows the differences and similarities between these huge systems to be brought out clearly. ${ }^{2}$

- Moreover, we can readily remix the ingredients to design new build systems with desired properties, for example, to combine the advantages of SHAKE and BAZEL. Writing this paper gave us the insights to combine dynamic dependencies and cloud build systems in a principled way; we evaluate the result in Section 7.

- We use the presented abstractions to more clearly explain details from the original SHAKE paper (Sections 5.2.2 and 7.2) and develop new cloud build features, which are already in use in industry and in the GHC build system (Sections 7.4 and 7.5).

In short, instead of seeing build systems as unrelated points in space, we now see them as locations in a connected landscape, leading to a better understanding of what they do and how they compare, and making it easier to explore other points in the landscape. While we steer clear of many engineering aspects of real build systems, in Section 8 , we discuss these aspects in the context of the presented abstractions. The related work is covered in Section 9.

This paper is an extended version of an earlier conference paper (Mokhov et al., 2018). The key changes compared to the earlier version are (i) we added further clarifications and examples to Section 3, and in particular, Section 3.8 is entirely new; (ii) Sections 4 and 5 are based on the material from the conference paper but have been substantially expanded to include further details and examples, as well as completely new material such as Section 5.2.2; (iii) Section 7 is completely new; (iv) Sections 8.1, 8.6, 8.7, 8.8, and 8.9 are almost entirely new, and Section 8.3 has been revised. The new material focuses on our experience and various important practical considerations, hence justifying the "and Practice" part of the paper title.

\section{Background}

Build systems automate the execution of repeatable tasks, at a scale from individual users up to large organisations. In this section, we explore the design space of build systems, using four examples: MAKE (Feldman, 1979), ShaKe (Mitchell, 2012), BAZEL (Google, 2016), and EXCEL (De Levie, 2004). We have carefully chosen these four to illustrate the various axes on which build systems differ; we discuss many other notable examples of build systems, and their relationships, in Sections 6 and 9.

1 EXCEL appears very different to the others but, seen through the lens of this paper, it is very close.

2 All our models are executable and are available on Hackage as build-1.0. 


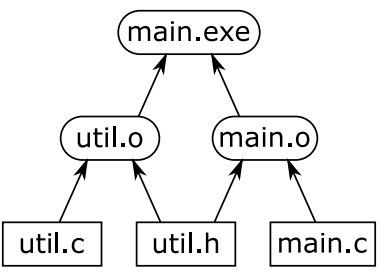

(a) Task dependency graph

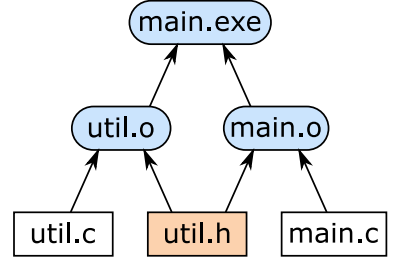

(b) Full rebuild

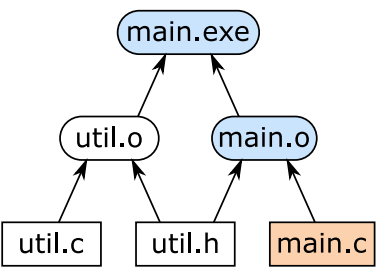

(c) Partial rebuild

Fig. 1. A task dependency graph and two build scenarios. Input files are shown in rectangles, intermediate, and output files are shown in rounded rectangles. Modified inputs and files that are rebuilt are highlighted.

\subsection{The venerable MАKE: Static dependencies and file modification times}

$\mathrm{MAKE}^{3}$ was developed more than 40 years ago to automatically build software libraries and executable programs from source code. It uses makefiles to describe tasks - often referred to as build rules - and their dependencies, in a simple textual form. For example:

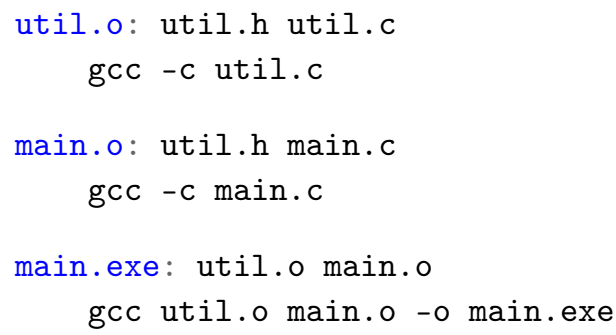

The above makefile lists three tasks: (i) compile a utility library comprising files util.h and util.c into util.o by executing ${ }^{4}$ the command gcc -c util.c, (ii) compile the main source file main.c into main.o, and (iii) link object files util.o and main.o into the executable main.exe. The makefile contains the complete information about the task dependency graph, which is shown in Figure 1(a).

If the user runs MAKE specifying main.exe as the desired output, MAKE will build util.o and main.o, in any order (or even in parallel) since these tasks are independent, and then main.exe. If the user modifies util.h and runs MAKE again, it will perform a full rebuild, because all three tasks transitively depend on util.h, as illustrated in Figure 1(b). On the other hand, if the user modifies main.c then a partial rebuild is sufficient: util.o does not need to be rebuilt, since its inputs have not changed, see Figure 1(c). Note that if the dependency graph is acyclic, then each task needs to be executed at most once. Cyclic task dependencies are typically not allowed in build systems, although there are rare exceptions, see Section 8.5.

The fewer tasks are executed in a partial rebuild, the better. To be more specific, consider the following property, which is essential for build systems; indeed, it is their raison d'être:

3 There are numerous implementations of MAKE and none comes with a formal specification. In this paper, we use a simple approximation to a real MAKE that you might find on your machine.

4 In this example, we pretend gcc is a pure function for the sake of simplicity. In reality, there are multiple versions of gcc. To account for this, the actual binary for gcc is often also listed as a dependency, along with any supporting binaries, or standard libraries (such as stdio.h), that are used by gcc. 
Definition (Minimality). A build system is minimal if it executes tasks at most once per build, and only if they transitively depend on inputs that changed since the previous build.

This property is tightly linked to build system correctness, which we will be ready to define in Section 3.6; for now, we will use minimality as a guiding principle when exploring the design space of build systems.

To achieve minimality, MAKE relies on two main ideas: (i) it uses file modification times to detect which files changed, ${ }^{5}$ and (ii) it constructs a task dependency graph from the information contained in the makefile and executes tasks in a topological order. For a more concrete description, see Sections 4.1 and 6.2.

\subsection{EXCEL: Dynamic dependencies at the cost of minimality}

EXCEL is a build system in disguise. Consider the following simple spreadsheet.
A1: 10
$\mathrm{B} 1: \mathrm{A} 1+\mathrm{A} 2$
A2: 20

There are two input cells $A 1$ and $A 2$, and a single task that computes the sum of their values, producing the result in cell B1. If either of the inputs change, EXCEL will recompute B1.

Unlike MAKE, EXCEL does not need to know all task dependencies upfront. Indeed, some dependencies may change dynamically during computation. For example:

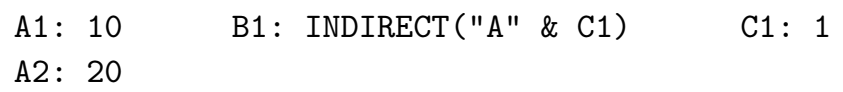

The formula in B1 uses the INDIRECT function, which takes a string and returns the value of the cell with that name. The string evaluates to "A1", so B1 evaluates to 10 . However, the dependencies of the formula in $\mathrm{B} 1$ are determined by the value of $\mathrm{C} 1$, so it is impossible to compute the dependency graph before the build starts. In this particular example, the value of $\mathrm{C} 1$ is a constant, but it might instead be the result of a long computation chain so its value will only become available during the build.

To support dynamic dependencies, EXCEL's calculation engine (Microsoft, 2011) is significantly different from MAKE. EXCEL arranges the cells into a linear sequence, called the calc chain. During the build, EXCEL processes cells in the calc-chain sequence, but if computing a cell $C$ requires the value of a cell $D$ that has not yet been computed, EXCEL aborts computation of $C$, moves $D$ before $C$ in the calc chain, and resumes the build starting with D. When a build is complete, the resulting calc chain respects all the dynamic dependencies of the spreadsheet. When an input value or formula is changed, EXCEL uses the final calc chain from the previous build as its starting point so that, in the common case where changing an input value does not change dependencies, there are no aborts. Notice that build always succeeds regardless of the initial calc chain (barring truly circular dependencies); the calc chain is just an optimisation. We refer to this algorithm as restarting and discuss it in more detail in Sections 4.2 and 6.3.

5 Technically, you can fool MAKE by altering the modification time of a file without changing its content, for example, using the command touch. MAKE is therefore minimal only under the assumption that you do not do that. 


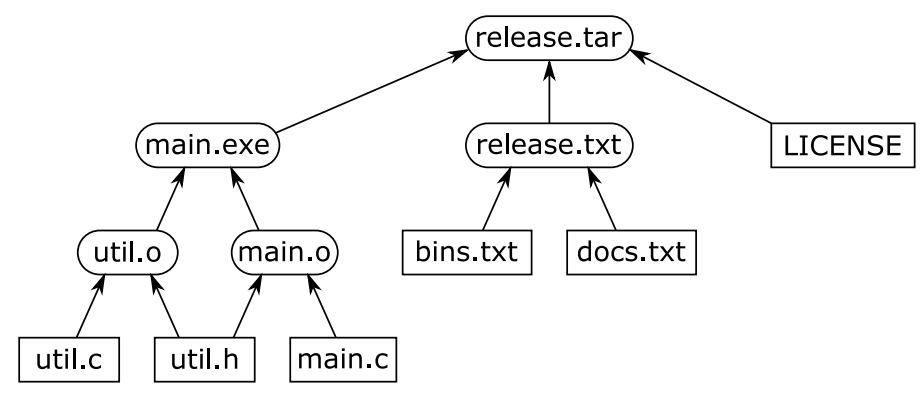

(a) Dependency graph produced after the previous build.

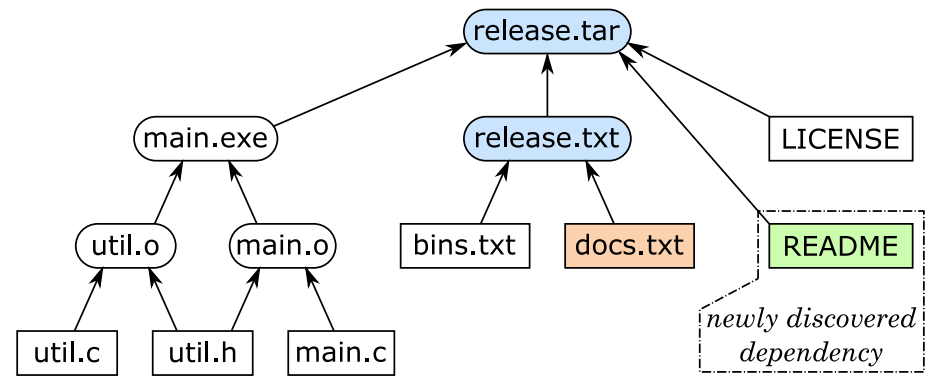

(b) The input file docs.txt was modified, hence we rebuild release.txt and release.tar, discovering a new dependency README in the process.

Fig. 2. Dynamic dependencies example: create README and add it to the list of release documents docs.txt.

Dynamic dependencies complicate minimality. In the above example, B1 should only be recomputed if $\mathrm{A} 1$ or $\mathrm{C} 1$ change, but not if (say) $\mathrm{A} 2$ changes; but these facts are not statically apparent. In practice, EXCEL implements a conservative approximation to minimality: it recomputes a formula if (i) the formula statically mentions a changed cell, or (ii) the formula uses a function like INDIRECT whose dependencies are not statically visible, or (iii) the formula itself has changed.

Item (iii) in the above list highlights another distinguishing feature of EXCEL: it is selftracking. Most build systems only track changes of inputs and intermediate results, but EXCEL also tracks changes in the tasks themselves: if a formula is modified, EXCEL will recompute it and propagate the changes. Self-tracking is uncommon in software build systems, where one often needs to manually initiate a full rebuild even if just a single task has changed. We discuss self-tracking further in Section 8.8.

\subsection{SHAKE: Dynamic dependencies without remorse}

SHAKE was developed to solve the issue of dynamic dependencies (Mitchell, 2012) without sacrificing the minimality requirement.

Building on the MAKE example from Section 2.1, we add the following files whose dependencies are shown in Figure 2(a):

- LICENSE is an input text file containing the project license. 


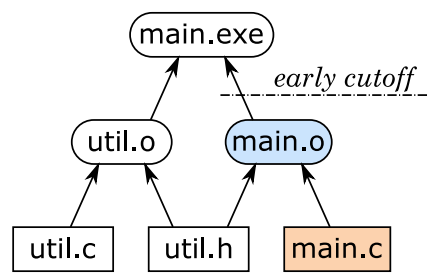

Fig. 3. An early cutoff example: if a comment is added to main.c, the rebuild is stopped after detecting that main.o is unchanged, since this indicates that main.exe and its dependents do not need to be rebuilt.

- release.txt lists all release files. This file is produced by concatenating input text files bins.txt and docs.txt, which list binary and documentation files of the project.

- release.tar is the archive built by executing the command tar on the release files.

The dependencies of release.tar are not known statically: they are determined by the content of release.txt, which might not even exist before the build. Makefiles cannot express such dependencies, requiring workarounds such as build phases, which are known to be problematic (Mokhov et al., 2016). In SHAKE, we can express the rule for release.tar as:

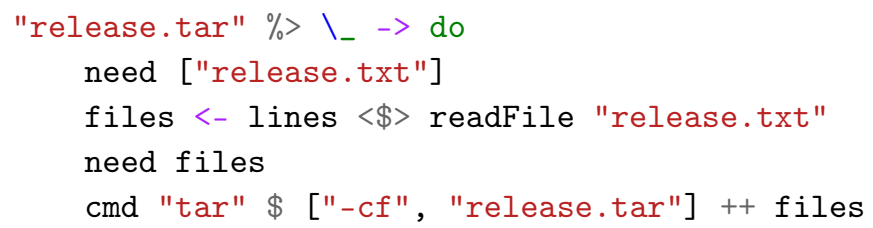

We first declare the static dependency on release.txt, then read its content (a list of files) and depend on each listed file, dynamically. Finally, we specify the command to produce the resulting archive. Crucially, the archive will only be rebuilt if one of the dependencies (static or dynamic) has changed. For example, if we create another documentation file README and add it to docs.txt, SHAKE will appropriately rebuild release.txt and release.tar, discovering the new dependency, see Figure 2(b).

SHAKE's implementation is different from both MAKE and EXCEL in two aspects. First, to decide which files need to be rebuilt, it stores the dependency graph that is constructed during the previous build (instead of just file modification times or a linear chain). This idea has a long history, going back to incremental (Demers et al., 1981), adaptive (Acar et al., 2002), and self-adjusting computations - see Acar et al. (2007) and Section 9. Second, instead of aborting and deferring the execution of tasks whose newly discovered dependencies have not yet been built (as EXCEL does), SHAKE suspends their execution until the dependencies are brought up to date. We refer to this task scheduling algorithm as suspending, see a further discussion in Section 4.3 and a concrete implementation in Section 6.4.

SHAKE also supports the early cutoff optimisation, which is illustrated in Figure 3. When it executes a task and the result is unchanged from the previous build, it is unnecessary to execute the dependent tasks, and hence SHAKE can stop a build earlier. Not all build systems support early cutoff: SHAKE and BAZEL (introduced below) do, but MAKE and EXCEL do not; see Section 5.1 for an explanation of why. 


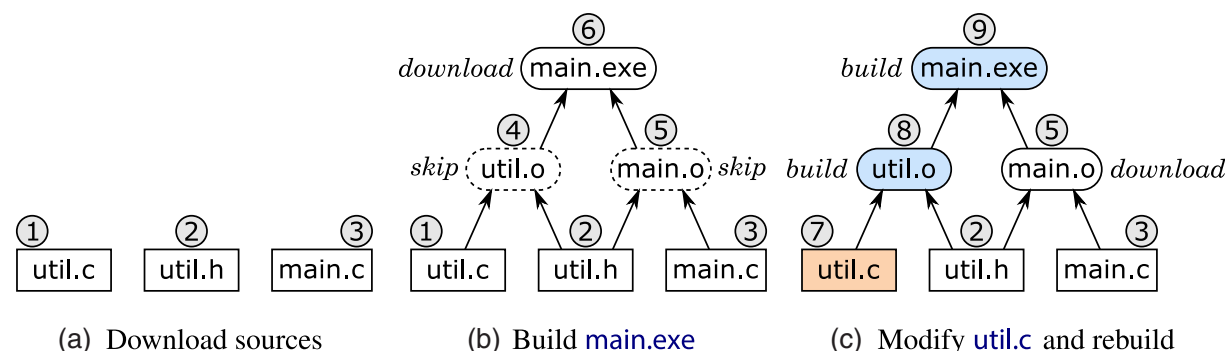

Fig. 4. A cloud build example: (a) download sources, (b) build main.exe by downloading it from the cloud and skipping intermediate files (only their hashes are needed), (c) modify util.c and rebuild main.exe, which requires building util.o (nobody has compiled util.c before) and downloading main.o (it is needed for linking main.exe). File hashes are shown in circles, and non-materialised intermediates in dashed rounded rectangles.

\subsection{BAZEL: A cloud build system}

When build systems are used by large teams, different team members often end up executing exactly the same tasks on their local machines. A cloud build system can speed up builds dramatically by sharing build results among team members. Furthermore, cloud build systems can support shallow builds that materialise only end build products locally, leaving all intermediates in the cloud.

Consider the example in Figure 4. The user starts by downloading the sources, whose content hashes are (for simplicity) 1, 2, and 3, and requests to build main.exe, see Figure 4(a) and (b). By looking up the global history of all previous builds, ${ }^{6}$ the build system finds that someone has already compiled these exact sources before, and the resulting files util.o and main.o had hashes 4 and 5, respectively. Similarly, the build system finds that the hash of the resulting main.exe was 6 and downloads the actual binary from the cloud storage - it must be materialised, because it is the end build product.

In Figure 4(c), the user modifies the source file util.c, thereby changing its hash from 1 to 7 . The cloud lookup of the new combination \{util.c, util.h\} fails, which means that nobody has ever compiled it. The build system must therefore build util.o, materialising it with the new hash 8 . The combination of hashes of util.o and main.o has not been encountered before either, thus the build system first downloads main.o from the cloud and then builds main.exe by linking the two object files. When the build is complete, the results can be uploaded to the cloud for future reuse by other team members.

BAZEL is one of the first openly available cloud build systems. As of writing, it is not possible to express dynamic dependencies in user-defined build rules; however, some of the predefined build rules require dynamic dependencies and the internal build engine can cope with them using a restarting task scheduler, which is similar to that of EXCEL but does not use the calc chain. BAZEL is not minimal in the sense that it may restart a task multiple times as new dependencies are discovered and rebuilt, but it supports the early cutoff optimisation. Note that, in practice, the cost of duplicate work due to the use of a restarting scheduler may often be just a small fraction of the overall build cost (Section 4.3).

\footnotetext{
6 In practice, old entries are regularly evicted from the cloud storage, as further discussed in Section 8.4.
} 
Table 1. Build system differences

\begin{tabular}{llllccr}
\hline Build system & $\begin{array}{l}\text { Persistent build } \\
\text { information }\end{array}$ & Scheduler & Dependencies & Minimal & Cutoff & Cloud \\
\hline MAKE & $\begin{array}{l}\text { File modification } \\
\text { times }\end{array}$ & Topological & Static & Yes & No & No \\
EXCEL & $\begin{array}{l}\text { Dirty cells, } \\
\text { calc chain }\end{array}$ & Restarting & Dynamic & No & No & No \\
SHAKE & $\begin{array}{l}\text { Previous dependency } \\
\text { graph } \\
\text { Cloud cache, } \\
\text { BAZEL }\end{array}$ & Suspending & Dynamic & Yes & Yes & No \\
& Restarting & Dynamic* & No & Yes & Yes \\
\hline
\end{tabular}

*At present, user-defined build rules cannot have dynamic dependencies.

To support cloud builds, BAZEL maintains (i) a content-addressable cache that maps the hash of a file's content to the actual content of that file; (ii) a memo table that records all executed build commands with their input and output file hashes. The memo table allows the build engine to bypass the execution of a task, by predicting the hash of the result from the hashes of its dependencies; then the content-addressable cache allows the engine to download the result (if needed) based on the result hash. Further details and a concrete implementation will be provided in Sections 5.3 and 6.5.

\subsection{Summary}

We summarise differences between four discussed build systems in Table 1. The column 'persistent build information' refers to the information that build systems persistently store between builds:

- MAKE stores file modification times, or rather, it relies on the file system to do that.

- EXCEL stores one dirty bit per cell and the calc chain from the previous build.

- SHAKE stores the dependency graph discovered in the previous build, annotated with file content hashes for efficient checking of file changes.

- BAZEL stores the content-addressable cache and the history of all previous build commands annotated with file hashes. This information is shared among all users.

In this paper, we elucidate which build system properties are consequences of specific implementation choices (stored metadata and task scheduling algorithm), and how one can obtain new build systems with desired properties by recombining parts of existing implementations. As a compelling example, in Section 6.5, we demonstrate how to combine the advantages of SHAKE and BAZEL.

\section{Build systems, abstractly}

We have introduced a number of components and characteristics of build systems: tasks, dependencies, early cutoff, minimality, etc. It is easy to get confused. To make all this more 
concrete, this section presents executable abstractions that can express all the intricacies of build systems discussed in Section 2 and allow us to construct complex build systems from simple primitives. Specifically, we present the task and build abstractions in Sections 3.2 and 3.3, respectively. Sections 4, 5, and 6 scrutinise the abstractions further and provide concrete implementations for several build systems.

\subsection{Common vocabulary for build systems}

Keys, values, and the store. The goal of any build system is to bring up to date a store that implements a mapping from keys to values. In software build systems, the store is the file system, the keys are filenames, and the values are file contents. In EXCEL, the store is the worksheets, the keys are cell names (such as A1), and the values are numbers, strings, etc., displayed as the cell contents. Many build systems use hashes of values as compact summaries with a fast equality check.

Input, output, and intermediate values. Some values must be provided by the user as input. For example, main.c can be edited by the user who relies on the build system to compile it into main.o and subsequently main.exe. End build products, such as main.exe, are output values. All other values (in this case main.o) are intermediate; they are not interesting for the user but are produced in the process of turning inputs into outputs.

Persistent build information. As well as the key/value mapping, the store also contains information maintained by the build system itself, which persists from one invocation of the build system to the next - its "memory".

Task description. Any build system requires the user to specify how to compute the new value for one key, using the (up-to-date) values of its dependencies. We call this specification the task description. For example, in EXCEL, the formulae of the spreadsheet constitute the task description; in MAKE, the rules in the makefile are the task description.

Build system. A build system takes a task description, a target key, and a store, and returns a new store in which the target key and all its dependencies have up-to-date values.

We model a build system concretely, as a Haskell program. To that end, Figure 5 provides the type signatures for all key abstractions introduced in the paper. For example, Store $\mathrm{i} \mathrm{k} \mathrm{v}$ is the type of stores, with several associated functions (getValue, etc.). We use $\mathrm{k}$ as a type variable ranging over keys, $v$ for values, and $i$ for the persistent build information. Figure 6 lists standard library definitions.

\subsection{The task abstraction}

Our first main abstraction is for task descriptions:

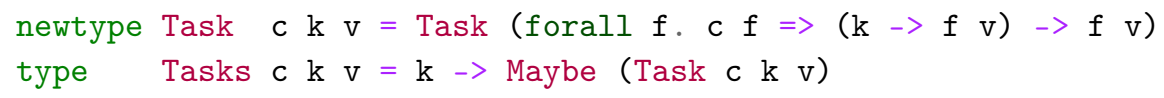

Here c stands for constraint, such as Applicative (Section 3.4 explains why we need it). A Task describes a single build task, while Tasks associates a Task with every non-input key; input keys are associated with Nothing. The highly abstracted type Task describes 


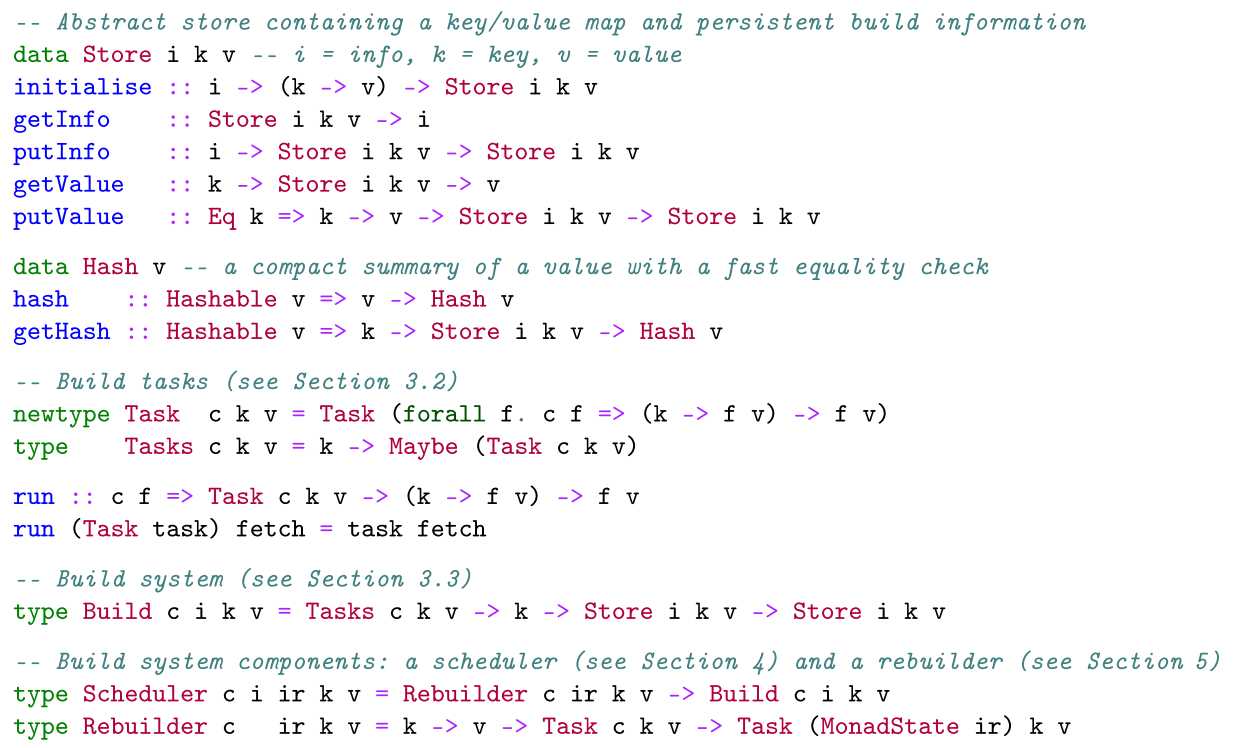

Fig. 5. Type signatures of key build systems abstractions.

how to build a value given a way to build its dependencies and is best explained by an example. Consider this EXCEL spreadsheet:
A1: 10
$\mathrm{B} 1: \mathrm{A} 1+\mathrm{A} 2$
A2: 20
$\mathrm{B} 2: \mathrm{B} 1 * 2$

Here, cell A1 contains the value 10, cell B1 contains the formula A1 + A2, etc. We can represent the formulae of this spreadsheet with the following task description:

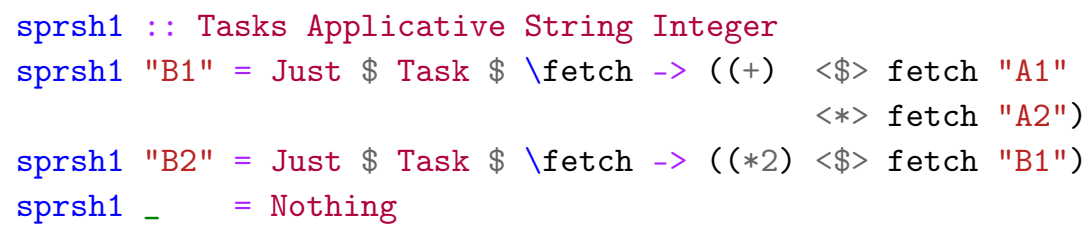

We instantiate keys $\mathrm{k}$ with String, and values $\mathrm{v}$ with Integer. (Real spreadsheet cells would contain a wider range of values, of course.) The task description sprsh1 embodies all the formulae of the spreadsheet, but not the input values. It pattern-matches on the key to see if it has a task description (in the EXCEL case, a formula) for it. If not, it returns Nothing, indicating that the key is an input. If there is a formula in the cell, it returns the Task to compute the value of the formula. Every task is given a callback fetch to find the value of any keys on which it depends. To run a Task, we simply apply the function it holds to a suitable callback (see the definition of the function run in Figure 5). 


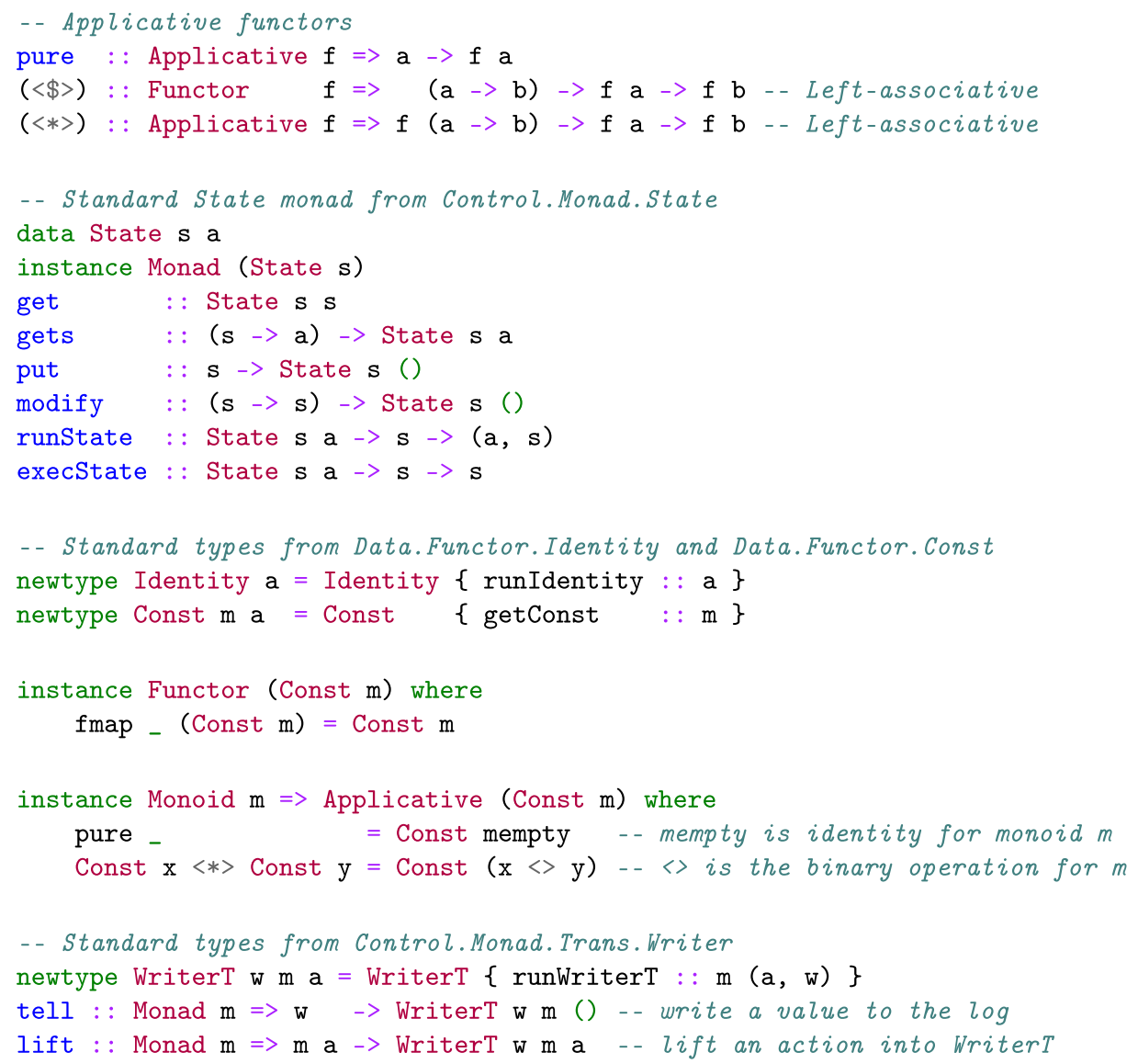

Fig. 6. Standard library definitions.

The code to "compute the value of a formula" in sprsh1 looks a bit mysterious because it takes place in an Applicative computation (McBride \& Paterson, 2008) - the relevant type signatures are given in Figure 6. We will explain why in Section 3.3. For now, we content ourselves with observing that a task description, of type Tasks c k v, is completely isolated from the world of compilers, calc chains, file systems, caches, and all other complexities of real build systems. It just computes a single output, using a callback (fetch) to find the values of its dependencies, and limiting side effects to those described by c.

\subsection{The build abstraction}

Next comes our second main abstraction - a build system:

type Build c i k v $=$ Tasks c k v $\rightarrow$ k $\rightarrow$ Store i k v $\rightarrow$ Store i k v

The signature is very straightforward. Given a task description, a target key, and a store, the build system returns a new store in which the value of the target key is up to date. What exactly does "up to date" mean? We answer that precisely in Section 3.6. 
Here is a simple build system:

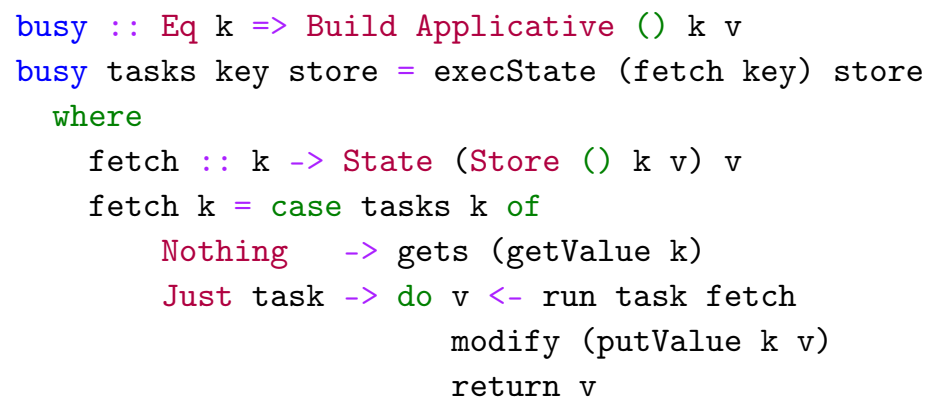

The busy build system defines the callback fetch that, when given a key, brings the key up to date in the store and returns its value. The function fetch runs in the standard Haskell State monad (Figure 6) initialised with the incoming store by execState. To bring a key $\mathrm{k}$ up to date, fetch asks the task description tasks how to compute its value. If tasks returns Nothing the key is an input, so fetch simply reads the result from the store. Otherwise fetch runs the obtained task to produce a resulting value $\mathrm{v}$, records the new key/value mapping in the store, and returns v. Notice that fetch passes itself to task as an argument, so the latter can use fetch to recursively find the values of k's dependencies.

Given an acyclic task description, the busy build system terminates with a correct result, but it is not a minimal build system (Definition 2.1). Since busy has no memory $(i=())$, it cannot keep track of keys it has already built and will therefore busily recompute the same keys again and again if they have multiple dependents. We will develop much more efficient build systems in Section 6.

Nevertheless, busy can easily handle the example task description sprsh 1 from the previous Section 3.2. In the GHCi session below, we initialise the store with A1 set to 10 and all other cells set to 20 .

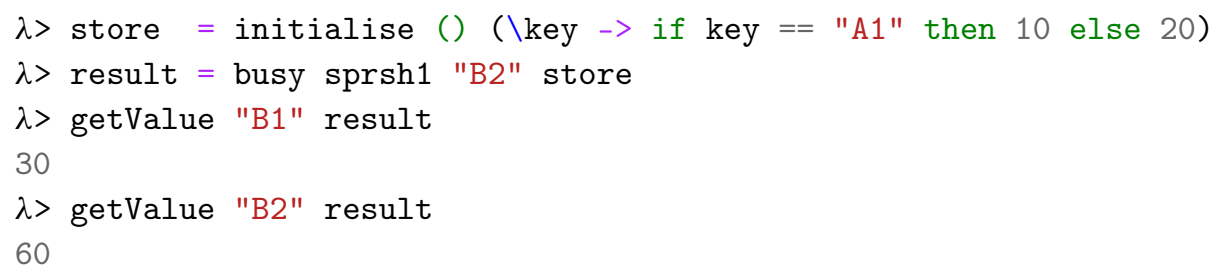

As we can see, busy built both B2 and its dependency B1 in the correct order (if it had built B2 before building B1, the result would have been $20 * 2=40$ instead of $(10+20) * 2=$ $60)$. As an example showing that busy is not minimal, imagine that the formula in cell B2 was $B 1+B 1$ instead of B1 * 2. This would lead to calling fetch "B1" twice - once per occurrence of B1 in the formula - and each call would recompute the formula in B1.

To avoid the recomputation, busy can keep the set of processed keys in the state monad (in addition to the store), treat processed keys as inputs in the getValue branch of the fetch callback, and include the key $\mathrm{k}$ into the set of processed keys in the putValue branch. This eliminates unnecessary work within a single build, but the next build needs to 
recursively recompute all target's dependencies again even if no inputs changed. To save work between builds, it is necessary to store some build information i persistently.

\subsection{The need for polymorphism in task}

The previous example illustrates why the Task abstraction is polymorphic in $f$. Recall its definition from Section 3.2:

$$
\text { newtype Task c k v }=\text { Task (forall f. c f } \Rightarrow>(k->f v)->f v)
$$

The busy build system instantiates $f$ to State (Store $i k v$ ), so that fetch : : k $->f v$ can side-effect the Store, thereby allowing successive calls to fetch to communicate with one another.

We really, really want Task to be polymorphic in $\mathrm{f}$. Given one task description $\mathrm{T}$, we want to explore many build systems that can build $\mathrm{T}$ - and we will do so in Section 6. As we shall see, each build system will use a different $f$, so the task description must not fix $f$.

But the task description cannot possibly work for any $f$ whatsoever; most task descriptions (e.g. sprsh1 in Section 3.2) require that $f$ satisfies certain properties, such as Applicative or Monad. That is why Task has the " $c f=>$ " constraint in its type, expressing that $f$ can only be instantiated by types that satisfy the constraint $c$ and, in exchange, the task has access to the operations of class c. So the type Task emerges naturally, almost inevitably. But now that it has emerged, we find that constraints c classify task descriptions in a very interesting, and practically useful, way:

- Task Applicative: In sprsh1, we needed only Applicative operations, expressing the fact that the dependencies between cells can be determined statically, that is, by looking at the formulae, without "computing" them (see Section 3.7).

- Task Monad: As we shall see in Section 3.5, a monadic task allows dynamic dependencies, in which a formula may depend on cell C, but which cell C depends on the value of another cell D. A simple example of a task with dynamic dependencies is EXCEL formula INDIRECT("A" \& C1) from Section 2.2.

- Task Functor is somewhat degenerate: a functorial task description cannot even use the application operator $\langle *\rangle$, which limits dependencies to a linear chain, as for example, in Docker containers (Hykes, 2013) (ignoring the recent multi-stage builds). It is interesting to note that, when run on such a task description, the busy build system will build each key at most once, thus partially fulfilling the minimality requirement 2.1. Alas, it still has no mechanism to decide which input keys changed since the previous build.

- Task Selective corresponds to task descriptions with conditional statements, for example, EXCEL formula IF(C1=1,B2,A2), where it is possible to statically overapproximate the set of task dependencies. Here Selective is a type class of selective applicative functors (Mokhov et al., 2019), which allows us to model build systems like DUNE (Jane Street, 2018) using the presented framework.

- Task MonadFail corresponds to monadic tasks that may fail. For example, the formula A1/A2 may fail due to division by zero. We will discuss this in Section 8.1. 
- Task MonadPlus, Task MonadRandom and their variants can be used for describing tasks with a certain type of non-determinism, as discussed in Section 8.3.

- Task (MonadState i) will be used in Section 6 to describe tasks that have read and write access to the persistently stored build information $i$.

\subsection{Monadic tasks}

As explained in Section 2.2, some task descriptions have dynamic dependencies, which are determined by values of intermediate computations. In our framework, such task descriptions correspond to the type Task Monad $\mathrm{k} v$. Consider this spreadsheet example:
A1: 10
$\mathrm{B} 1: \operatorname{IF}(\mathrm{C} 1=1, \mathrm{~B} 2, \mathrm{~A} 2)$
C1: 1
A2: 20
$\mathrm{B} 2: \operatorname{IF}(\mathrm{C} 1=1, \mathrm{~A} 1, \mathrm{~B} 1)$

Note that B1 and B2 statically form a dependency cycle, so MAKE would not be able to order the tasks topologically, but EXCEL, which uses dynamic dependencies, is perfectly happy. We can express this spreadsheet using our task abstraction as follows:

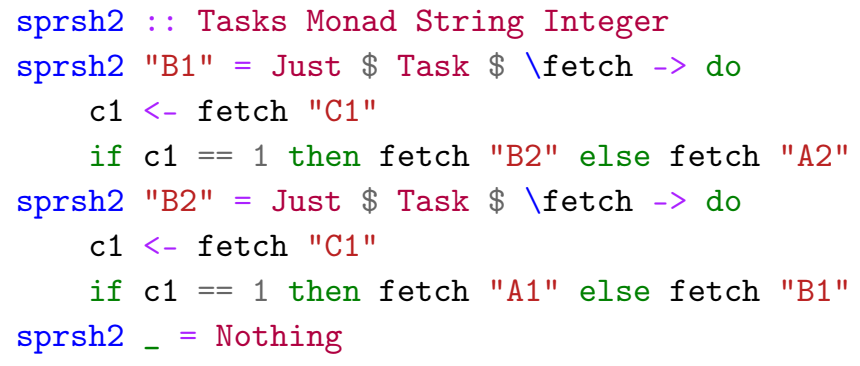

The big difference compared to sprsh1 is that the computation now takes place in a Monad, which allows us to extract the value of $\mathrm{c} 1$ and fetch different keys depending on whether or not $c 1==1$. Note that, in this example, one can statically determine the sets of possible dependencies of the formulae $\operatorname{IF}(C 1=1, B 2, A 2)$ and $\operatorname{IF}(C 1=1, A 1, B 1)$ but this cannot be done in general - recall the spreadsheet with the formula INDIRECT("A" \& C1) from Section 2.2, where the argument of the INDIRECT function is a string computed dynamically during the build. Such tasks can also be captured using Tasks Monad:

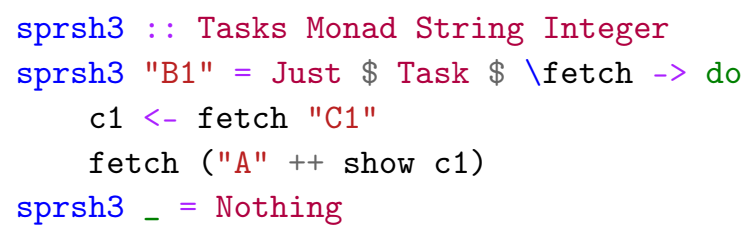

Since the busy build system introduced in Section 3.3 always rebuilds every dependency it encounters, it is easy for it to handle dynamic dependencies. For minimal build systems, however, dynamic dependencies, and hence monadic tasks, are much more challenging, as we shall see in Section 6. 


\subsection{Correctness of a build system}

We can now say what it means for a build system to be correct, something that is seldom stated formally. Our intuition is this: when the build system completes, the target key, and all its dependencies, should be up to date. What does "up to date" mean? It means that if we recompute the value of the key (using the task description and the final store), we should get exactly the same value as we see in the final store.

To express this formally, we need an auxiliary function compute that computes the value of a key in a given store without attempting to update any dependencies:

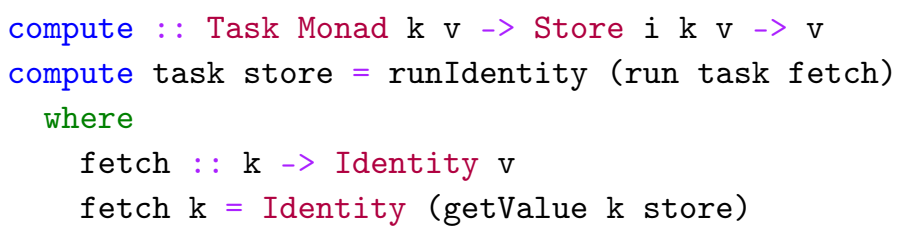

Here, we do not need any effects in the fetch callback to task, so we can use the standard Haskell Identity monad (Figure 6). This is another use of polymorphism in $f$, discussed in Section 3.4. The use of Identity just fixes the "impedance mismatch" between the function getValue, which returns a pure value $\mathrm{v}$, and the fetch argument of the task, which must return an $f \mathrm{v}$ for some $f$. To fix the mismatch, we wrap the result of getValue in the Identity monad and pass to the task. The result has type Identity v, which we unwrap with runIdentity.

Definition (Correctness). Suppose build is a build system, tasks is a build task description, key is a target key, store is an initial store, and result is the store produced by running the build system with parameters tasks, key, and store. Or, using the precise language of our abstractions:

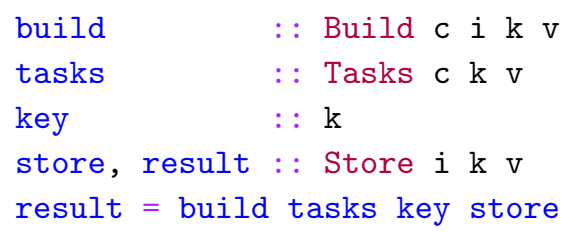

The keys that are reachable from the target key via dependencies fall into two classes: input keys and non-input keys, which we will denote by $I$ and $O$, respectively. Note that key may be in either of these sets, although the case when key is an input is degenerate: we have $I=\{$ key $\}$ and $O=\emptyset$.

The build result is correct if the following two conditions hold:

- result and store agree on inputs, that is, for all input keys $\mathrm{k} \in I$ :

getValue $\mathrm{k}$ result $==$ getValue $\mathrm{k}$ store.

In other words, no inputs were corrupted during the build. 
- The result is consistent with the tasks, that is, for all non-input keys $\mathrm{k} \in O$, the result of recomputing the corresponding task matches the value stored in the result:

$$
\text { getValue } \mathrm{k} \text { result }==\text { compute task result. }
$$

A build system is correct if it produces a correct result for any tasks, key, and store.

It is hard to satisfy the above definition of correctness given a task description with cycles. All build systems discussed in this paper are correct only under the assumption that the given task description is acyclic. This includes the busy build system introduced earlier: it will loop indefinitely given a cyclic tasks. Some build systems provide a limited support for cyclic tasks, see Section 8.5.

The presented definition of correctness needs to be adjusted for build systems that support non-deterministic tasks and shallow cloud builds, as will be discussed in Sections 8.3 and 8.4, respectively.

\subsection{Computing dependencies}

Earlier, we remarked that a Task Applicative could only have static dependencies. Usually, we would extract such static dependencies by (in the case of EXCEL) looking at the syntax tree of the formula. But a task description has no such syntax tree: as you can see in the definition of Task in Figure 5, a task is just a function, so all we can do is call it. Yet, remarkably, we can use the polymorphism of a Task Applicative to find its dependencies without doing any of the actual work. Here is the code:

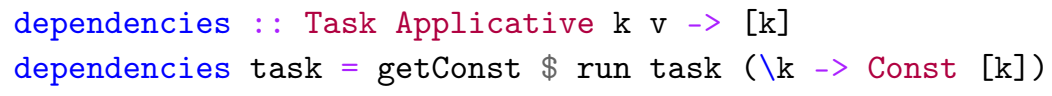

Here Const is a standard Haskell type defined in Figure 6. We instantiate $f$ to Const $[k]$. So a value of type $f \mathrm{v}$, or in this case Const [k] v, contains no value $\mathrm{v}$, but does contain a list of keys of type $[\mathrm{k}]$ which we use to record dependencies. The fetch callback that we pass to task records a single dependency; and the standard definition of Applicative for Const (which we give in Figure 6) combines the dependencies from different parts of the task. Running the task with $f=$ Const $[\mathrm{k}]$ will thus accumulate a list of the task's dependencies - and that is what dependencies does:

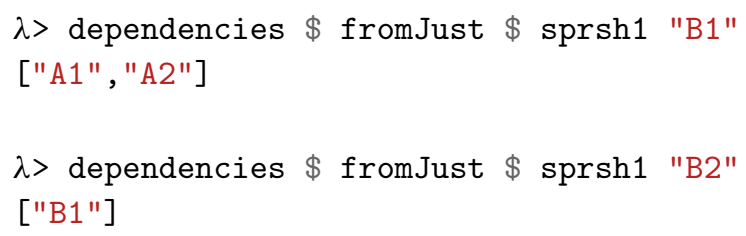

Notice that these calls to dependencies do no actual computation (in this case, spreadsheet arithmetic). They cannot: we are not supplying a store or any input numbers. So, through the wonders of polymorphism, we are able to extract the dependencies of the spreadsheet formula, and to do so efficiently, simply by running its code in a different Applicative! This is not new, for example see Capriotti \& Kaposi (2014), but it is 
extremely cool. We will see a practical use for dependencies when implementing applicative build systems, see Section 6.2.

So much for applicative tasks. What about monadic tasks with dynamic dependencies? As we have seen in Section 2.3, dynamic dependencies need to be tracked too. This cannot be done statically; notice that we cannot apply the function dependencies to a Task Monad because the Const functor has no Monad instance. We need to run a monadic task on a store with concrete values, which will determine the discovered dependencies. Accordingly, we introduce the function track - a combination of compute and dependencies that computes both the resulting value and the list of its dependencies (key/value pairs) in an arbitrary monadic context $\mathrm{m}$. We need this function to be polymorphic over $\mathrm{m}$, because each build system will execute tasks in its own monad, as we shall see in Section 6.

Here is an implementation of track based on the standard Haskell WriterT monad transformer (Liang et al., 1995), whose main types are listed in Figure 6:

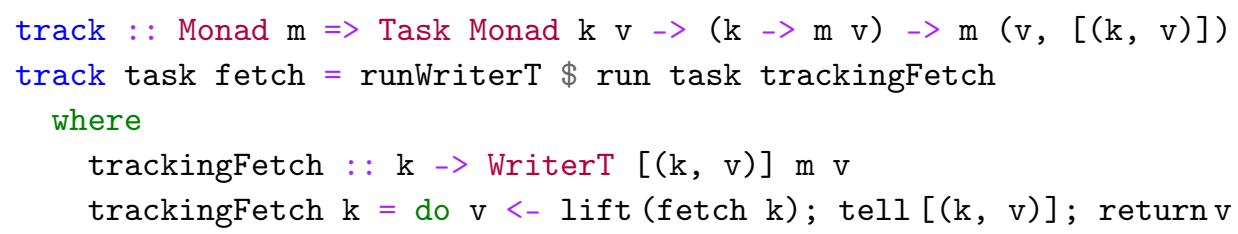

This function uses the WriterT transformer for recording additional information - a list of key/value pairs $[(\mathrm{k}, \mathrm{v})]$ - when executing a task in an arbitrary monad $\mathrm{m}$. We substitute the given fetch with a trackingFetch that, in addition to fetching a value, tracks the corresponding key/value pair. The task returns a value of type WriterT [ $(\mathrm{k}, \mathrm{v})] \mathrm{m} v$, which we unwrap with runWriterT. We will use track when implementing monadic build systems with dynamic dependencies, see Section 6.4 .

Here we show an example of tracking monadic tasks when $\mathrm{m}=\mathrm{IO}$, by defining a corresponding fetchIO of type String -> IO Integer, which allows us to demonstrate the dynamic nature of monadic dependencies in GHCi.

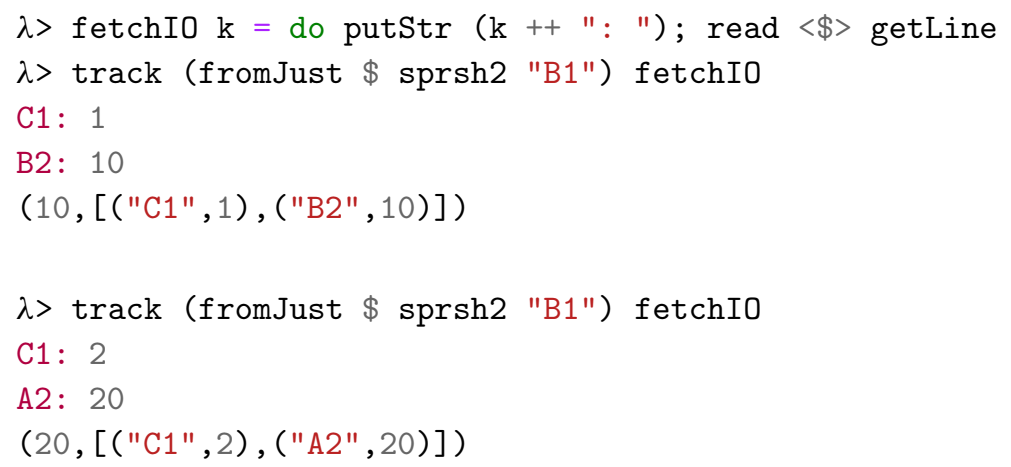

As expected, the dependencies of the cell B1 from sprsh2 (see the spreadsheet in Section 3.5) are determined by the value of $C 1$, which in this case is obtained by reading from the standard input using fetchIO. 


\subsection{Examples of tasks}

In this section, we give examples of tasks whose definitions involve different constraints on the computation context: Functor, Applicative, Monad, and MonadState s. The purpose of these examples is to continue building the intuition behind the Task abstraction and prepare the reader for richer types of tasks that will appear in Sections 6 and 8.

We start with one of the favourite examples for functional programmers - the Fibonacci sequence $F_{n}=F_{n-1}+F_{n-2}$ :

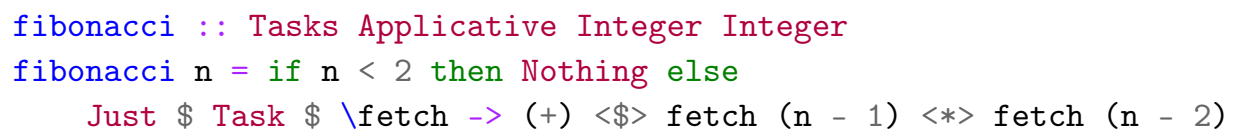

Here the keys $n<2$ are input parameters, and one can obtain the usual Fibonacci sequence by picking $F_{0}=0$ and $F_{1}=1$, respectively. Any minimal build system will compute the sequence with memoisation, that is, without recomputing the same value twice.

Dependencies of elements of the Fibonacci sequence are known statically, hence we can express it using Tasks Applicative and benefit from static dependency analysis (Section 3.7):

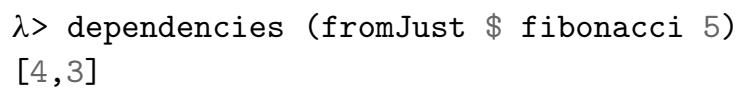

Interestingly, the Ackermann function - a famous example of a function that is not primitive recursive - cannot be expressed as an applicative task, because it needs to perform an intermediate recursive call to determine the value of one of its dependencies $A(m, n-1)$ :

$$
A(m, n)= \begin{cases}n+1 & \text { if } m=0 \\ A(m-1,1) & \text { if } m>0 \text { and } n=0 \\ A(m-1, A(m, n-1)) & \text { if } m>0 \text { and } n>0\end{cases}
$$

We therefore use Tasks Monad to express this function, binding the dynamic dependency to variable index:

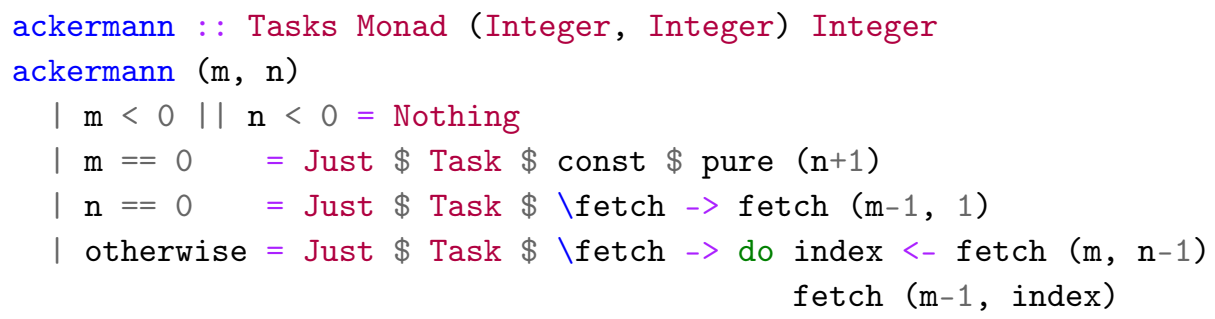

Functorial tasks are less common than applicative and monadic, but there is a classic example too - the Collatz sequence, where given an initial value $c_{0}$, we calculate the next value 
$c_{n}$ from $c_{n-1}$ either by dividing $c_{n-1}$ by 2 (if it is even) or multiplying it by 3 and adding 1 (if it is odd):

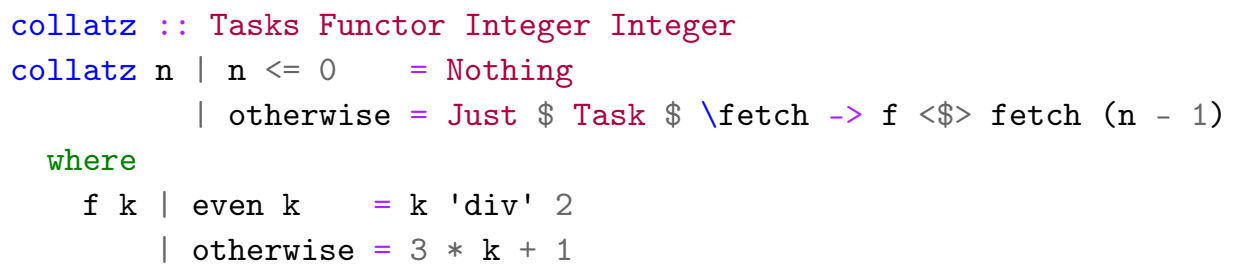

Functorial tasks correspond to computations with a linear dependency chain. For example, computing the element $c_{8}$ of the Collatz sequence starting from $c_{0}=6$ leads to the following dependency chain: $c_{0}=6 \rightarrow 3 \rightarrow 10 \rightarrow 5 \rightarrow 16 \rightarrow 8 \rightarrow 4 \rightarrow 2 \rightarrow 1=c_{8}$.

Collatz sequence is a good example of the early cutoff optimisation (Section 2.3): if we recompute $c_{8}$ starting from a different initial value $c_{0}=40$, the resulting computation will have a large overlap with the previous one: $c_{0}=40 \rightarrow 20 \rightarrow 10 \rightarrow 5 \rightarrow 16 \rightarrow 8 \rightarrow 4 \rightarrow$ $2 \rightarrow 1=c_{8}$. We can therefore stop the recomputation after just two steps, since $c_{2}=10$ has not changed.

Note that we can statically extract even more precise dependency information from functorial tasks compared to applicative tasks. Indeed, we statically know that a Task Functor has exactly one dependency:

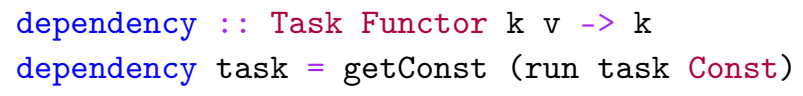

The Tasks abstraction allows us to express pure functions in a way that is convenient for their memoisation and incremental recomputation (see Section 9.3 for a discussion on memoisation). If we furthermore need to share computation results via a cloud cache, we can use Tasks (MonadState s) that will play an important role in Section 6. Intuitively, by making a shared state of type s available to a task, we give it the abilities to lookup and update cached computation results using the MonadState methods get and modify. For example, below we implement a cloud version of the Ackermann task that uses a Cache of type Map (Integer, Integer) Integer for sharing results of known Ackermann values.

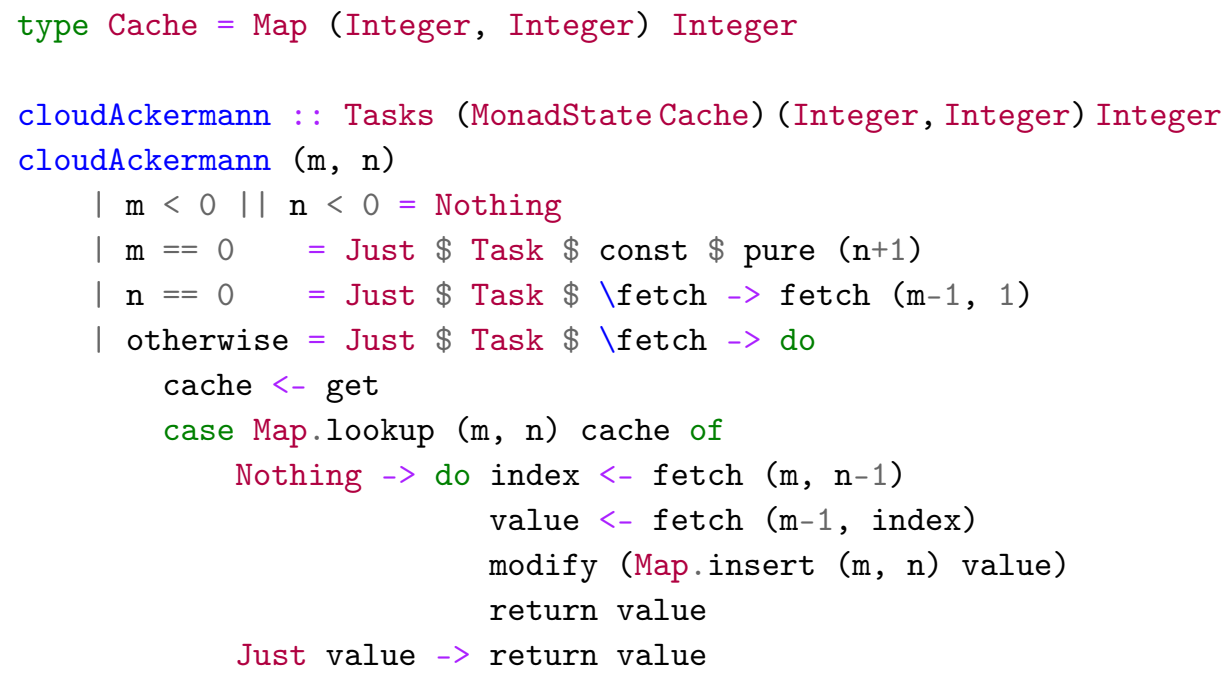


The main case $(m>0 \wedge n>0)$ starts by looking up the pair of indices $(m, n)$ in the cache. If the cache has Nothing, we calculate the resulting value as before and modify the cache accordingly; otherwise, if we have a cache hit, we return the obtained value immediately, skipping the actual calculation and thus potentially saving a large amount of work. Indeed, you do not want to recompute $A(4,2)=2^{65,536}-3$ unnecessarily; all of its 19,729 decimal digits have already been helpfully computed, for example, see Kosara (2008). We will use MonadState tasks in our models of cloud build systems in Section 6.

\section{Schedulers}

The focus of this paper is on a variety of implementations of Build c i k v, given a usersupplied implementation of Tasks $\mathrm{c} \mathrm{k} \mathrm{v}$. That is, we are going to take Tasks as given from now on and explore variants of Build: first, abstractly (in this section and in Section 5) and then concretely in Section 6.

As per the definition of minimality (Section 2.1), a minimal build system must rebuild only out-of-date keys and at most once. The only way to achieve the "at most once" requirement while producing a correct build result (Section 3.6) is to build all keys in an order that respects their dependencies.

We have emboldened two different aspects above: the part of the build system responsible for scheduling tasks in the dependency order (a "scheduler") can be cleanly separated from the part responsible for deciding whether a key needs to be rebuilt (a "rebuilder"). In this section, we discuss schedulers, leaving rebuilders for Section 5.

Section 2 introduced three different task schedulers that decide which tasks to execute and in what order; see the "Scheduler" column of Table 1 in Section 2.5. The following subsections explore the properties of the three schedulers, and possible implementations.

\subsection{Topological scheduler}

The topological scheduler precomputes a linear order of tasks, which when followed ensures dependencies are satisfied, then executes the required tasks in that order. Computing such a linear order is straightforward - given a task description and a target key, first find the (acyclic) graph of the key's dependencies, then compute a topological order. Taking the MAKE example from Figure 1, we might compute the following order:

1. main.o

2. util.o

3. main.exe

Given the dependencies, we could have equally chosen to build util.o first, but main.exe must come last.

The advantage of this scheme is simplicity - compute an order, then execute tasks in that order. In addition, any missing keys or dependency cycles can be detected from the graph and reported to the user before any work has commenced.

The downside of this approach is that it requires the dependencies of each task in advance. As we saw in Section 3.7, we can only extract dependencies from an applicative 
task, which requires the build system to choose $c=$ Applicative, ruling out dynamic dependencies.

\subsection{Restarting scheduler}

To handle dynamic dependencies, we cannot precompute a static order - we must interleave running tasks and ordering tasks. One approach is just to build tasks in an arbitrary order, and if a task calls fetch on an out-of-date key dep, abort the task and build dep instead. Returning to the example from Figure 1, we might build the tasks as follows:

1. main.exe (abort because it depends on util.o which is out of date)

2. main.o

3. util.o

4. main.exe (restart from scratch, completing successfully this time)

We start with main.exe (an arbitrary choice), but discover it depends on main.o, so instead start building main.o. Next, we choose to build util.o (again, arbitrarily), before finally returning to main.exe that now has all its dependencies available and completes successfully.

This approach works, but has a number of disadvantages. Firstly, it requires a technical mechanism to abort a task, which is easy in our theoretical setting with Task (see an implementation in Section 6.3) but leads to engineering concerns in the real world. Secondly, it is not minimal in the sense that a task may start, do some meaningful work, and then abort, repeating that same work when restarted.

As a refinement, to reduce the number of aborts (often to zero), EXCEL records the discovered task order in its calc chain and uses it as the starting point for the next build (Section 2.2). BAZEL's restarting scheduler does not store the discovered order between build runs; instead, it stores the most recent task dependency information from which it can compute a linear order. Since this information may become outdated, BAZEL may also need to abort a task if a newly discovered dependency is out of date.

\subsection{Suspending scheduler}

An alternative approach, utilised by the busy build system (Section 3.3) and SHAKE, is to simply build dependencies when they are requested, suspending the currently running task when needed. Using the example from Figure 1, we would build

- main.exe (suspended)

$\hookrightarrow$ main.o

- main.exe (resumed then suspended again)

$\hookrightarrow$ util.o

- main.exe (completed)

We start building main.exe first as it is the required target. We soon discover a dependency on main.o and suspend the current task main.exe to build main.o, then resume and suspend again to build util.o, and finally complete the target main.exe. 
This scheduler (when combined with a suitable rebuilder) provides a minimal build system that supports dynamic dependencies. In our model, a suspending scheduler is easy to write - it makes a function call to compute each dependency. However, a more practical implementation is likely to build multiple dependencies in parallel, which then requires a more explicit task suspension and resumption. To implement suspension, there are two standard approaches:

- Blocking threads or processes: this approach is relatively easy, but can require significant resources, especially if a large number of tasks are suspended. In languages with cheap green threads (e.g. Haskell), the approach is more feasible and it was the original approach taken by SHAKE.

- Continuation-passing style (Claessen, 1999) can allow the remainder of a task to be captured, paused, and resumed later. Continuation passing is efficient, but requires the build script to be architected to allow capturing continuations. SHAKE currently uses this approach.

While a suspending scheduler is theoretically optimal, in practice, it is better than a restarting scheduler only if the cost of avoided duplicate work outweighs the cost of suspending tasks. Note, furthermore, that the cost of duplicate work may often be just a fraction of the overall build cost.

\section{Rebuilders}

A build system can be split into a scheduler (as defined in Section 4) and a rebuilder. Suppose the scheduler decides that a key should be brought up to date. The next question is: does any work need to be done, or is the key already up to date? Or, in a cloud build system, do we have a cached copy of the value we need?

While Section 2 explicitly listed the schedulers, the rebuilders were introduced more implicitly, primarily by the information they retain to make their decisions. From the examples, we have looked at, we see four fundamental rebuilders, each with a number of tweaks and variations within them.

\subsection{A dirty bit}

The idea of a dirty bit is to have one piece of persistent information per key, saying whether the key is dirty or clean. After a build, all bits are set to clean. When the next build starts, anything that changed between the two builds is marked dirty. If a key and all its transitive dependencies are clean, the key does not need to be rebuilt. Taking the example from Figure 1(c), if main.c changes then it would be marked dirty, and main.o and main.exe would be rebuilt as they transitively depend on main.c.

EXCEL models the dirty-bit approach most directly, having an actual dirty bit associated with each cell, marking the cell dirty if the user modifies it. It also marks dirty all cells that (transitively) depend on the modified cell. EXCEL does not record dynamic dependencies 
of each cell; instead, it computes a static over-approximation - it is safe for it to make more cells dirty than necessary, but not vice versa. The over-approximation is as follows: a cell is marked dirty (i) if its formula statically refers to a dirty cell, or (ii) if the formula calls a volatile function like INDIRECT whose dependencies cannot be guessed from the formula alone. The over-approximation is clear for INDIRECT, but it is also present for IF, where both branches are followed even though dynamically only one is used.

MAKE uses file modification times and compares files to their dependencies, which can be thought of as a dirty bit which is set when a file is older than its dependencies. The interesting property of this dirty bit is that it is not under the control of MAKE; rather it is existing file system information that has been repurposed. Modifying a file automatically clears its dirty bit and automatically sets the dirty bit of the files depending on it (but not recursively). Note that MAKE requires that file timestamps only go forward in time, which can be violated by backup software.

With a dirty bit, it is possible to achieve minimality (Section 2.1). However, to achieve early cutoff (Section 2.3), it would be important to clear the dirty bit after a computation that did not change the value and make sure that keys that depend on it are not rebuilt unnecessarily. For EXCEL, this is difficult because the dependent cells have already been recursively marked dirty. For MAKE, it is impossible to mark a file clean and at the same time not mark the files that depend on it dirty. MAKE can approximate early cutoff by not modifying the result file, and not marking it clean, but then it will be rebuilt in every subsequent build.

A dirty-bit rebuilder is useful to reduce memory consumption, and in the case of MAKE, to integrate with the file system. However, as the examples show, in constrained environments where a dirty bit is chosen, it is often done as part of a series of compromises.

It is possible to implement a dirty-bit rebuilder that is minimal and supports early cutoff. To do so, the build system should start with all inputs that have changed marked dirty, then a key must be rebuilt if any of its direct dependencies are dirty, marking the key dirty only if the result has changed. At the end of the build, all dirty bits must be cleared. This approach only works if all targets are rebuilt each time because clearing dirty bits of keys that are not transitive dependencies of current targets will cause them to incorrectly not rebuild subsequently. To avoid resetting the dirty bit, it is possible to use successive execution numbers, which ultimately leads to an approach we call verifying step traces in Section 5.2.2.

\subsection{Verifying traces}

An alternative way to determine if a key is dirty is to record the values/hashes of dependencies used last time, and if something has changed, the key is dirty and must be rebuilt - in essence, keeping a trace which we can use to verify existing values. Taking the example from Figure 4(c), we might record that the key util.o (at hash 8) depended on the keys util.c (at hash 7) and util.h (at hash 2). Next time round, if the scheduler decides that it is time for util.o to be rebuilt and all keys still have the same hashes as in the recorded trace, there is nothing to do, and we can skip rebuilding. If any of the hashes is different, we rebuild util.o and record a trace with the new values. 
For traces, there are two essential operations - adding a new trace to the trace store and using the trace store to determine if a key needs rebuilding. Assuming a store of verifying traces VT k v, the operations are:

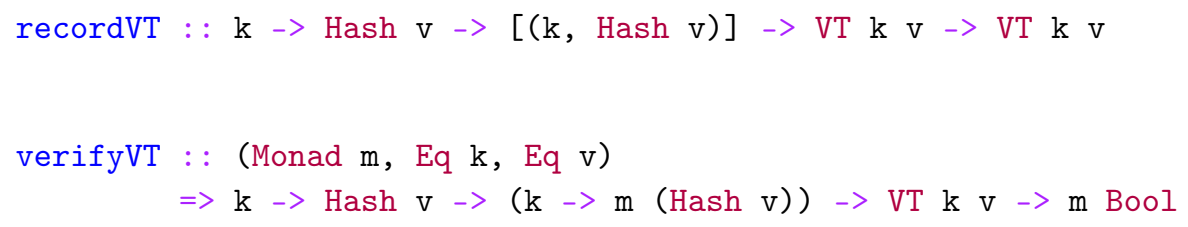

Rather than storing (large) values $\mathrm{v}$, the verifying trace VT can store only hashes of those values, with type Hash v. Since the verifying trace persists from one build to the next - it constitutes the build system's "memory" - it is helpful for it to be of modest size. After successfully building a key, we call recordVT to add a record to the current VT, passing the key, the hash of its value, and the list of hashes and dependencies.

More interestingly, to verify whether a key needs rebuilding we use verifyVT, supplying the key, the hash of its current value, a function for obtaining the hash of the post-build value of any key (using a scheduling strategy as per Section 4), and the existing trace store VT. The result will be a Bool where True indicates that the current value is already up to date, and False indicates that it should be rebuilt.

The most complex argument of verifyVT is a function fetchHash : : $k->m$ (Hash v) to obtain the hash of the post-build value of any key. With an applicative task, fetchHash will be called on the statically known task dependencies. However, with a monadic task, the dependencies are not known from the task alone, they are only recorded from previous executions stored in VT. If the build system has two traces for a given key $k$, they will both request the same dependency first, since Task Monad is deterministic. However, based on that first result, they may then request different subsequent dependencies using fetchHash. A curious result is that, for suspending schedulers (Section 4.3) in many cases, the actual build steps are performed as a consequence of checking if a key needs rebuilding!

A verifying trace and other types of traces discussed in this section support dynamic dependencies and minimality; furthermore, all traces except for deep traces (Section 5.4) support the early cutoff optimisation (Section 2.3).

\subsubsection{Trace representation}

One potential implementation would be to record all arguments passed to recordVT in a list and verify by simply checking if any list item matches the information passed by verifyVT. Concretely, in our implementations from Section 6, traces are recorded as lists of:

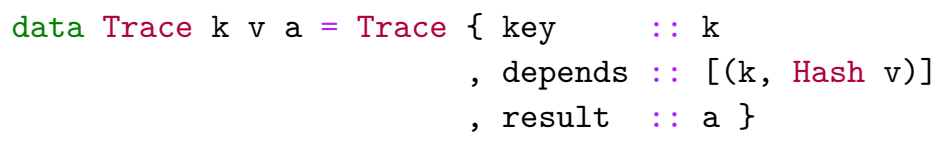

Where $\mathrm{a}$ is Hash $\mathrm{v}$ for verifying traces (and $\mathrm{v}$ for constructive traces, discussed later in Section 5.3). A real system is highly likely to use a more optimised implementation. 
The first optimisation is that any system using Applicative dependencies can omit the dependency keys from the Trace, since they can be recovered from the key field (Section 3.7).

The next optimisation is that there is only very minor benefit from storing more than one Trace per key. Therefore, verifying traces can be stored as Map k (Trace k v (Hash v)), where the initial $\mathrm{k}$ is the key field of Trace, thus making verifyVT much faster. Note that storing only one Trace per key means that if the dependencies of a key change but the resulting value does not, and then the dependencies change back to what they were before, there will be no valid Trace available and the key will therefore have to be rebuilt, whereas a complete list of all historical traces would allow the rebuilding to be skipped. On the other hand, bounding the number of Trace structures by the number of distinct keys, regardless of how many builds are executed, is a useful property.

\subsubsection{Verifying step traces}

The SHAKE build system and the associated paper - see Section 2.3.3 in Mitchell (2012) use a different trace structure, called verifying step traces, which stores less data than verifying traces, and has slightly different early cutoff semantics. Rather than storing the Hash $\mathrm{v}$ for each dependency, it instead stores built time and changed time for each key, and a list of dependency keys (without the hashes). The resulting StepTrace type resembles

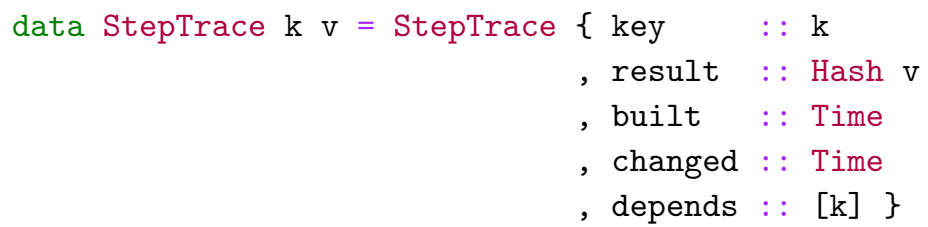

The built field is when the key last rebuilt. The changed field is when the result last changed - if the last build changed the value, it will be equal to built, otherwise it will be older. The function recordVT consults the previous step traces to know whether to keep the previous changed value or change it to built. The function verifyVT is a bit more subtle; given a key $\mathrm{k}$ and the hash of its current value $\mathrm{h}$, it performs the following steps:

- Find the latest (with respect to the field built) step trace matching $\mathrm{k}$. If it does not exist, return False: $\mathrm{k}$ was never built before and cannot be verified.

- If $h$ does not equal the result field of the trace, return False: the current $k$ 's value was changed externally and thus needs rebuilding.

- For each key d in depends:

- Make sure $d$ is up to date, suspending the current task if needed;

- If d's latest changed time is greater than k's built time, return False.

- Return True: the current k's value is up to date.

This approach preserves minimality and early cutoff. A variant with only one Time field would lose early cutoff and indeed corresponds quite closely to MAKE. Furthermore, the Time stamp only needs to record which execution of the build is running, so every key built in the same run can share the same Time value - it just needs to be monotonically increasing between runs. 


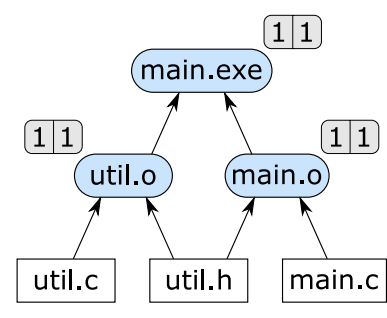

(a) Initial full build

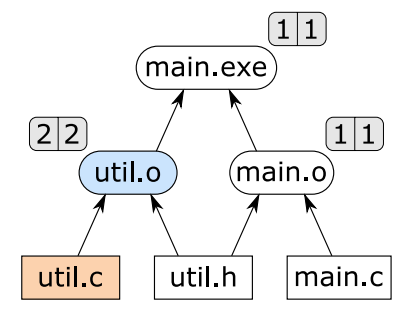

(b) Change util.c, build util.o

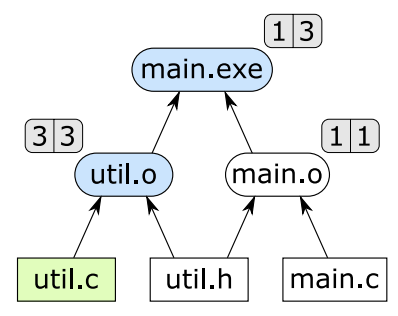

(c) Restore util.c, build main.exe

Fig. 7. An example of verifying step traces. The small rectangles show the changed (left) and built (right) timestamps of each non-input key in the trace store.

This optimisation is useful, at least in the case of SHAKE, to save space. A typical cryptographic hash takes up 32 bytes, while a key (in SHAKE) is an Int taking only 4 bytes. Furthermore, SHAKE permits values to be arbitrarily large and supports a custom value equality (two values can be bit-for-bit unequal but considered equal by SHAKE), hence Hash $\mathrm{v}$ is not a valid encoding. For applicative tasks, depends can be omitted, making the size of a StepTrace $O(1)$ instead of $O(n)$, where $n$ is the number of dependencies.

While verifying step traces are mostly an optimisation, there are some observable differences from verifying traces, as demonstrated by an example in Figure 7. We first make the full build: all keys get a built and changed of timestamp 1. Next, we change util.c and build util.o; the latter is changed as a result and hence both built and changed are increased to 2. Finally, we change util.c back to what it was originally and build main.exe. With verifying traces, the hashes of the dependencies of main.exe would be equal to the initial build and main.exe would not need rebuilding. With verifying step traces, the changed field of util.o would increase once more and main.exe would therefore be rebuilt. As shown in Figure 7(c), the changed field of main.exe remains 1, since the actual value is unchanged. Other than when building subsets of the targets, we are unaware of any other situation where verifying step traces are less powerful.

\subsection{Constructive traces}

A verifying trace records only hashes or timestamps, so that it can be small. In contrast, a constructive trace also stores the resulting value. Concretely, it records a list of Trace k v v, where Trace is as defined in Section 5.2.1. Once, we are storing the complete result, it makes sense to record many constructive traces per key and to share them with other users, providing cloud build functionality. We can access this additional information with these operations:

$$
\begin{aligned}
\operatorname{recordCT} & : \mathrm{k} \rightarrow \mathrm{v}->[(\mathrm{k}, \text { Hash } \mathrm{v})] \rightarrow \mathrm{CT} \mathrm{k} \mathrm{v} \rightarrow \mathrm{CT} \mathrm{k} \mathrm{v} \\
\text { constructCT } & ::(\text { Monad } \mathrm{m}, \text { Eq } \mathrm{k}, \text { Eq } \mathrm{v}) \\
& \Rightarrow \mathrm{k} \rightarrow(\mathrm{k}->\mathrm{m}(\text { Hash } \mathrm{v})) \rightarrow \text { CT } \mathrm{k} \mathrm{v} \rightarrow \mathrm{m}[\mathrm{v}]
\end{aligned}
$$

The function recordCT is similar to recordVT from Section 5.2, but instead of just passing the hash of the resulting value, we pass the actual value. The function verifyVT has 
been replaced with constructCT, which instead of taking the hash of the current value as input, returns a list of possible values as output - there may be more than one, because some build tools are non-deterministic, see Section 8.3.

Regardless of the chosen scheduler (Section 4), there are three cases to consider when using a rebuilder based on constructive traces:

- If constructCT returns the empty list of possible values, the key must be rebuilt.

- If the current value in the store matches one of the possible values, the build system can skip this key. Here, a constructive trace is used for verifying the current value.

- If the current value in the store does not match any possible value, we can use any of the possible values without doing any work to build it and copy it into the store.

Any Applicative build system using constructive traces, for example CloudBuild (Section 6.5), can index directly from the key and the hashes of its dependencies to the resulting value, for example, using a Map ( $k$, [Hash v]) v. Importantly, assuming the traces are stored on a central server, the client can compute the key and the hashes of its dependencies locally and then make a single call to the server to retrieve the result.

In practice, many cloud build systems store hashes of values in the trace store, that is, use Trace k v (Hash v) entries just like verifying traces and have a separate contentaddressable cache which associates hashes with their actual contents.

\subsection{Deep constructive traces}

Constructive traces always verify keys by looking at their immediate dependencies, which must have first been brought up to date, meaning that the time to verify a key depends on the number of transitive dependencies. A deep constructive trace optimises this process by only looking at the terminal input keys, ignoring any intermediate dependencies. The operations capturing this approach are the same as for constructive traces in Section 5.3, but we use the names recordDCT and constructDCT, where the underlying DCT representation need only record information about hashes of inputs, not intermediate dependencies.

Concretely, taking the example from Figure 1, to decide whether main.exe is out of date, a constructive trace would look at util.o and main.o (the immediate dependencies), whereas a deep constructive trace would look at util.c, util.h and main.c.

An advantage of deep constructive traces is that to decide if main.exe is up to date only requires consulting its inputs, not even considering util.o or main.o. Such a feature is often known as a shallow build, as discussed in Section 8.4.

There are two primary disadvantages of deep constructive traces:

- Tasks must be deterministic: If the tasks are not deterministic then it is possible to violate correctness, as illustrated by the example in Section 8.4, see Figure 12.

- No early cutoff: Deep constructive traces cannot support early cutoff (Section 2.3), since the results of intermediate computations are not considered.

Current build systems using deep constructive traces always record hashes of terminal input keys, but the technique also works if we skip any number of dependency levels (say $n$ levels). The input-only approach is the special case of $n=\infty$, and constructive traces are 
Table 2. Build systems à la carte

\begin{tabular}{lcccc}
\hline $\begin{array}{l}\text { Rebuilding } \\
\text { strategy }\end{array}$ & & $\begin{array}{c}\text { Topological } \\
\text { Section } 4.1\end{array}$ & $\begin{array}{c}\text { Restarting } \\
\text { Section } 4.2\end{array}$ & $\begin{array}{c}\text { Suspending } \\
\text { Section 4.3 }\end{array}$ \\
\hline Dirty bit & Section 5.1 & MAKE & EXCEL & - \\
Verifying traces & Section 5.2 & NINJA & - & SHAKE \\
Constructive traces & Section 5.3 & CLOUDBUILD & BAZEL & - \\
Deep constructive traces & Section 5.4 & BUCK & - & NIX \\
\hline
\end{tabular}

the special case of $n=1$. By picking values of $n$ in between we would regain some early cutoff, at the cost of losing such simple shallow builds, while still requiring determinism.

\section{Build systems, concretely}

In the previous sections, we discussed the types of build systems and how they can be broken down into two main components: a scheduler (Section 4) and a rebuilder (Section 5). In this section, we make this abstract distinction concrete by implementing a number of build systems as a composition of a scheduler and a rebuilder. The result can be summarised in Table 2, which tabulates 12 possible combinations, 8 of which are inhabited by existing build systems (we discuss these systems in Sections 2 and 9.1). Of the remaining four spots, all result in workable build systems. The most interesting unfilled spot in the table corresponds to a suspending scheduler composed with a constructive trace rebuilder. Such a build system would provide many benefits; we title it CLOUD SHAKE and explore further in Section 6.5.

\subsection{Concrete implementations}

We can define schedulers and rebuilders more concretely with the following types (Figure 5):

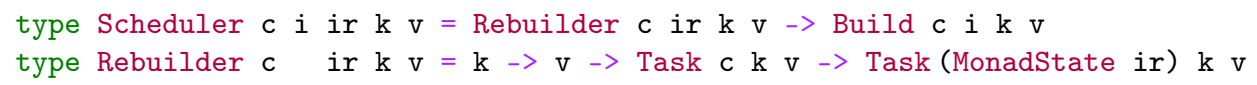

A Scheduler is a function that takes a Rebuilder and uses it to construct a Build system by choosing which keys to rebuild in which order. The Rebuilder makes use of the persistent build information ir, while the scheduler might augment that with further persistent information of its own, yielding $i$.

A Rebuilder takes three arguments: a key, its current value, and a Task that can (re)compute the value of the key if necessary. It uses the persistent build information ir (carried by the state monad) to decide whether to rebuild the value. If doing so is unnecessary, it returns the current value; otherwise, it runs the supplied Task to rebuild it. In both cases, it can choose to update the persistent build information ir to reflect what happened. So, a Rebuilder wraps a Task c k v, which unconditionally rebuilds the key, to make a Task (MonadState ir) $\mathrm{k} \mathrm{v}$, which rebuilds the key only if necessary, and does the necessary book-keeping. Note that the resulting Task is always monadic; static dependency analysis can be performed on the original Task Applicative if needed. 
The scheduler runs the Task returned by the rebuilder passing it a fetch callback that the task uses to find values of its dependencies. The callback returns control to the scheduler, which may in turn call the rebuilder again to bring another key up to date, and so on.

These two abstractions are the key to modularity: we can compose any scheduler with any rebuilder and obtain a correct build system. In this section, we will write a scheduler for each column of Table 2, and a rebuilder for each row; then compose them to obtain the build systems in the table's body.

\subsection{MAKE}

An implementation of MAKE using our framework is shown in Figure 8. As promised, its definition is just the application of a Scheduler, topological, to a Rebuilder, modTimeRebuilder. We discuss each component in turn, starting with the rebuilder.

The modTimeRebuilder uses the pair MakeInfo $\mathrm{k}=$ (now, modTimes) as persistent build information, carried by a state monad. This MakeInfo comprises the current time now : : Time and the map modTimes : : Map k Time of file modification times. We assume that the external system, which invokes the build system, updates MakeInfo reflecting any file changes between successive builds.

The rebuilder receives three arguments: a key, its current value, and the applicative task that can be used to rebuild the key if necessary. The rebuilder first decides if the key is dirty by consulting modTimes: if the key is not found, that must mean it has never been built before; otherwise, modTimeRebuilder can see if any of the task's dependencies (computed by dependencies) are out of date. If the key is dirty, we use run task to rebuild it and update the state with the new modification time of the key ${ }^{7}$; otherwise, we can just return the current value.

MAKE's scheduler, topological, processes keys in a linear order based on a topological sort of the statically known dependency graph (see Section 8.2 for parallel MAKE). Our definition in Figure 8 is polymorphic with respect to the type of build information i and is therefore compatible with any applicative rebuilder. The scheduler calls the supplied rebuilder on every key in the order and runs the obtained newTask to compute the newValue. Note that newTask has access only to the i part of the Store i k v, but the rest of the do block runs in the State (Store i k v) monad; we use the (unremarkable) helper function liftStore to fix the mismatch. The newTask finds values of the key's dependencies via the fetch callback, which is defined to directly read the store.

The pre-processing stage uses the function dependencies, defined in Section 3.7, to extract static dependencies from the provided applicative task. We compute the linear processing order by constructing the graph of keys reachable from the target via dependencies and performing the topological sort of the result. We omit implementation of textbook graph algorithms reachable and topSort, for example, see Cormen et al. (2001).

Note that the function dependencies can only be applied to applicative tasks, which restricts MAKE to static dependencies, as reflected in the type Build Applicative. Any other build system that uses the topological scheduler will inherit the same restriction.

7 The real MAKE relies on the file system to track file modification times, but we prefer to make this explicit in our model. 


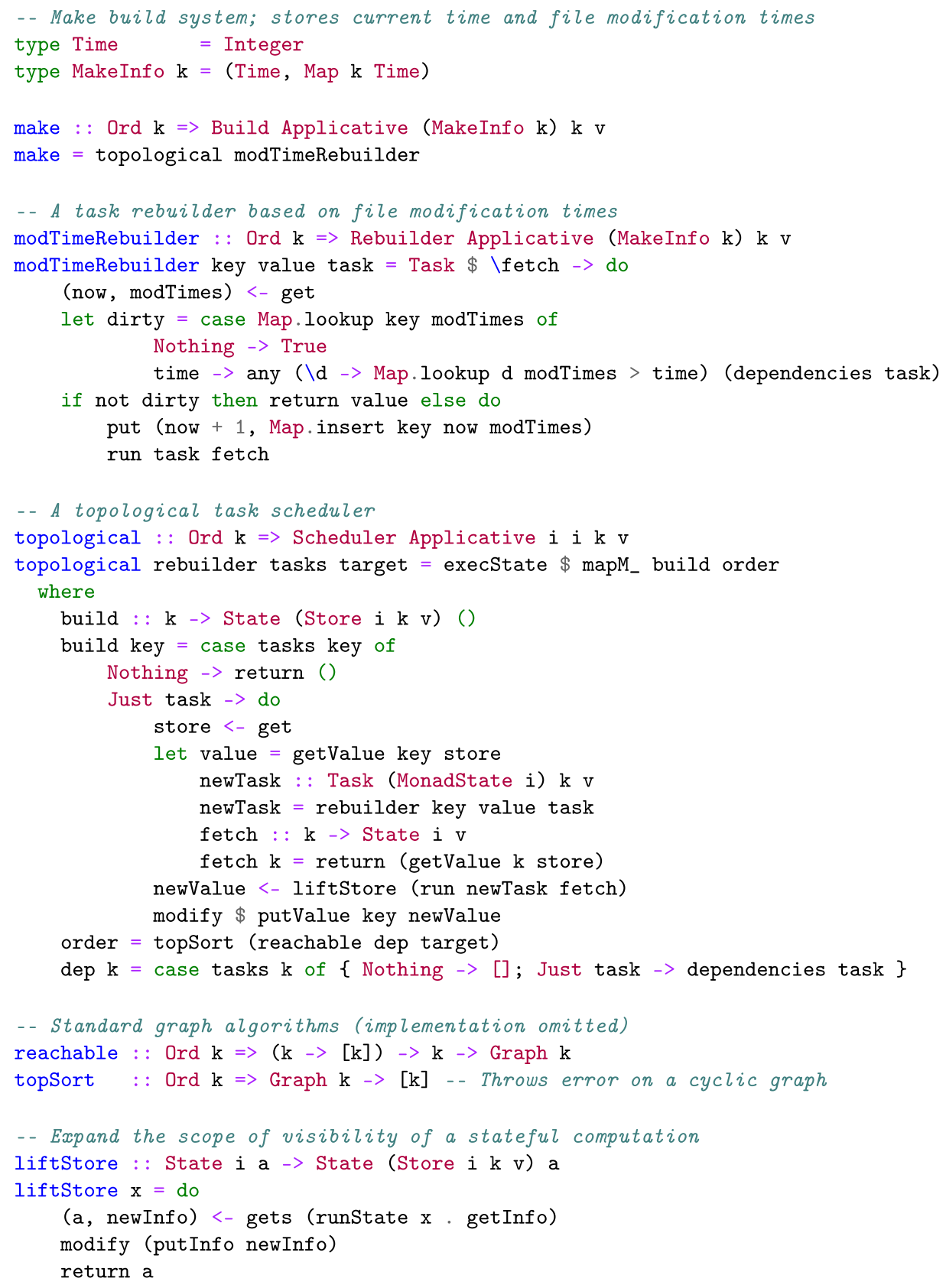

Fig. 8. An implementation of MAKE using our framework.

\subsection{EXCEL}

Our model of EXCEL uses the restarting scheduler and the dirtyBitRebuilder, see Figure 9. The persistent build information Excel Info $k$ is a pair of: (i) a map $k->$ Bool associating a dirty bit with every key and (ii) a calc chain of type $[\mathrm{k}]$ recorded from the previous build (Section 2.2). 


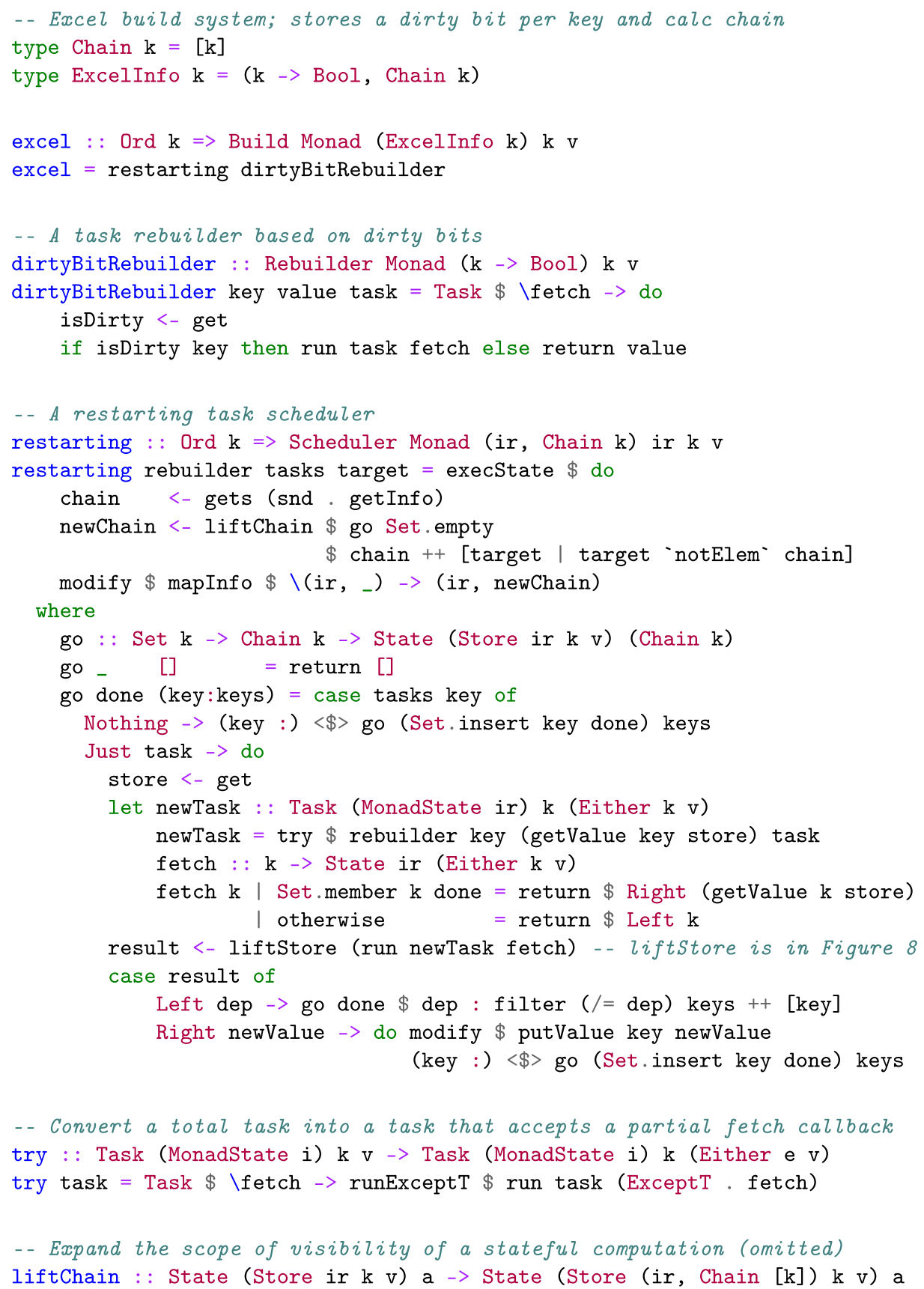

Fig. 9. An implementation of EXCEL using our framework.

The external system, which invokes EXCEL's build engine, is required to provide a transitively closed set of dirty bits. That is, if a cell is changed, its dirty bit is set, as well as the dirty bit of any other cell whose value might perhaps change as a result. It is OK to mark too many cells as dirty; but not OK to mark too few. 
The dirtyBitRebuilder is very simple: if the key's dirty bit is set, we run the task to rebuild the key; otherwise, we return the current value as is. Because the dirty cells are transitively closed, unlike MAKE's modTimeRebuilder, the dirtyBitRebuilder does not need to modify $i$ to trigger rebuilds of dependent keys.

EXCEL's restarting scheduler processes keys in the order specified by the calc chain. During the build, it constructs a newChain for the next build and maintains a set of keys done that have been processed. For each non-input key, the scheduler tries to rebuild it using a partial fetch callback that returns Either $\mathrm{k} v$ instead of $\mathrm{v}$. The callback is defined to fail with Left dep when asked for the value of a dependency dep that has not yet been processed (and hence may potentially be dirty); otherwise, it returns the current value of the dependency by looking it up in the store.

After the newTask is executed (using liftStore), there are two cases to consider:

- The newTask has failed, because one of its dependencies dep has not yet been processed. This indicates that the calculation chain from the previous build is incorrect and needs to be adjusted by moving the dep in front of the key, so that we can restart building the key after the dep is ready.

- The newTask succeeded. The resulting newValue is written to the store, the key is marked as done, and EXCEL continues to build the rest of the chain.

Note that the task returned by the rebuilder expects a total callback function and cannot be executed with the partial callback fetch. We fix the mismatch with the function try that relies on the standard monad transformer ExceptT (Liang et al., 1995). The helper liftChain is analogous to liftStore in Figure 8, so we omit its implementation.

\subsection{SHAKE}

Our model of SHAKE (Figure 10) stores verifying traces VT k v defined in Section 5.2 as persistent build information and is composed of the suspending scheduler and the vtRebuilder.

The rebuilder performs the verifyVT query to determine if the key is upToDate. If it is, the rebuilder simply returns the key's current value. Otherwise, it executes the task, obtaining both a newValue and the key's dynamic dependencies deps (see the definition of track in Section 3.7), which are subsequently recorded in the trace store using recordVT.

The suspending scheduler uses a recursive fetch callback, defined similarly to the busy build system (Section 3.3), that builds a given key, making sure not to duplicate work when called on the same key again in future. To achieve that, it keeps track of keys that have already been built in a set done : : Set k. Given a non-input key that has not yet been built, we use the supplied rebuilder to embed the build information $i$ into the task. We then execute the obtained newTask by passing it the fetch function as a callback for building dependencies: the newTask will therefore be suspended while its dependencies are being brought up to date. The newValue obtained by running the newTask is stored, and the key is added to the set done.

The fetch computation runs in the State (Store i k v, Set k) monad. To make MonadState $i$ access the $i$ inside the Store, we use the helper function liftRun (which uses a newtype to provide a MonadState instance that sees through into the Store). 


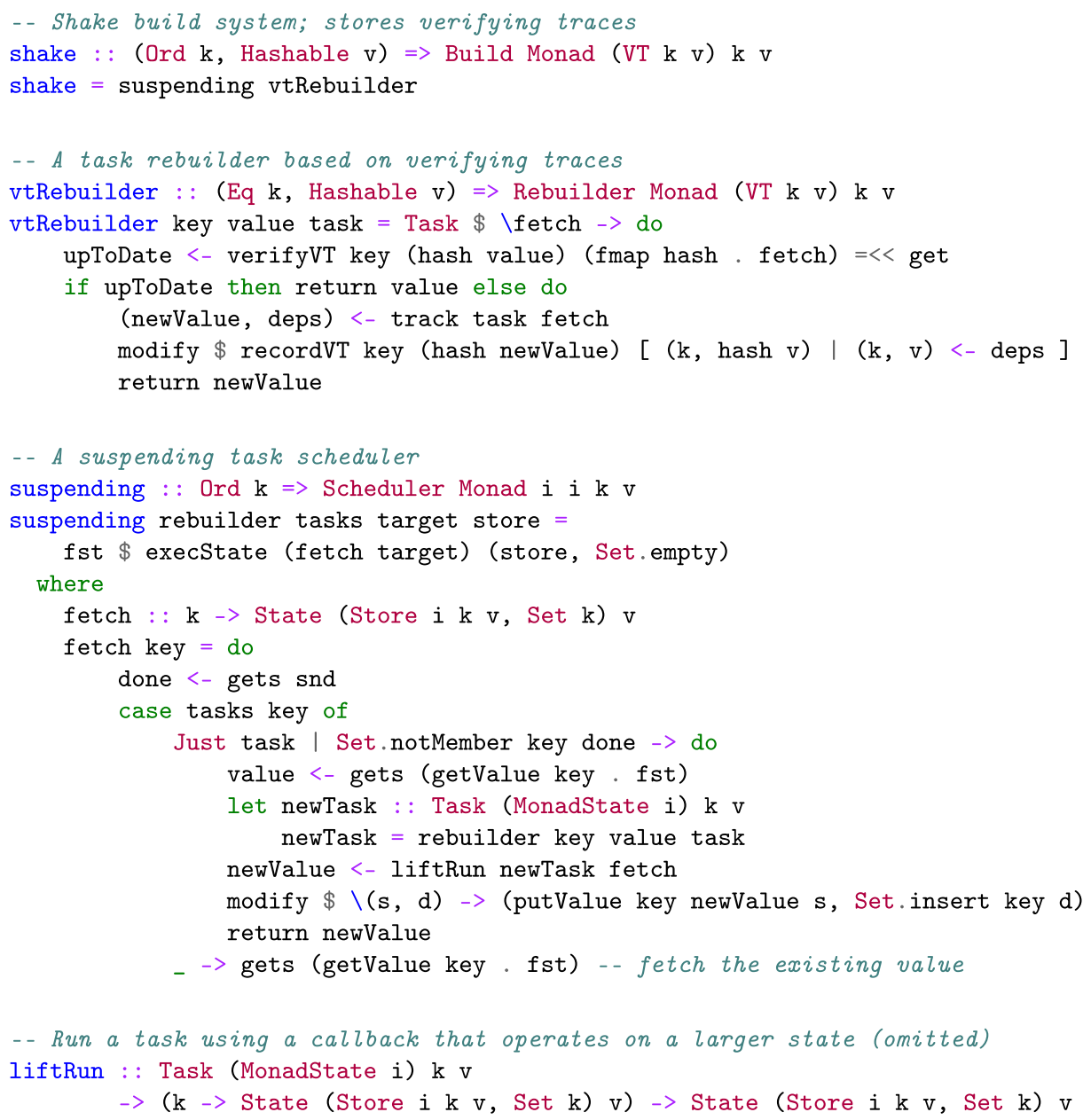

Fig. 10. An implementation of SHAKE using our framework.

As discussed in Section 5.2.2, SHAKE actually uses verifying step traces, but here we choose to focus on the more explicit verifying traces. We have implemented verifying step traces in our framework, and they compose with schedulers as you would hope.

\subsection{Cloud build systems: BAZel, ClOudBulld, Cloud ShAKe, BuCK and NiX}

Figure 11 shows our models of several cloud build systems. BAZEL, CLOUDBUILD, and Cloud SHAKE are based on constructive traces (Section 5.3), whereas BUCK and NIX use deep constructive traces (Section 5.4).

The implementation of ctRebuilder is analogous to that of vtRebuilder in Figure 10, but the verifyVT query is replaced with a more powerful query to constructCT that returns a list of suitable cachedValues by looking them up the cloud cache. If the current value is in the list, we can use it as is. Otherwise, if the list is non-empty, we can use an arbitrary cachedValue. Finally, if the cache has no suitable values, we fall back to 


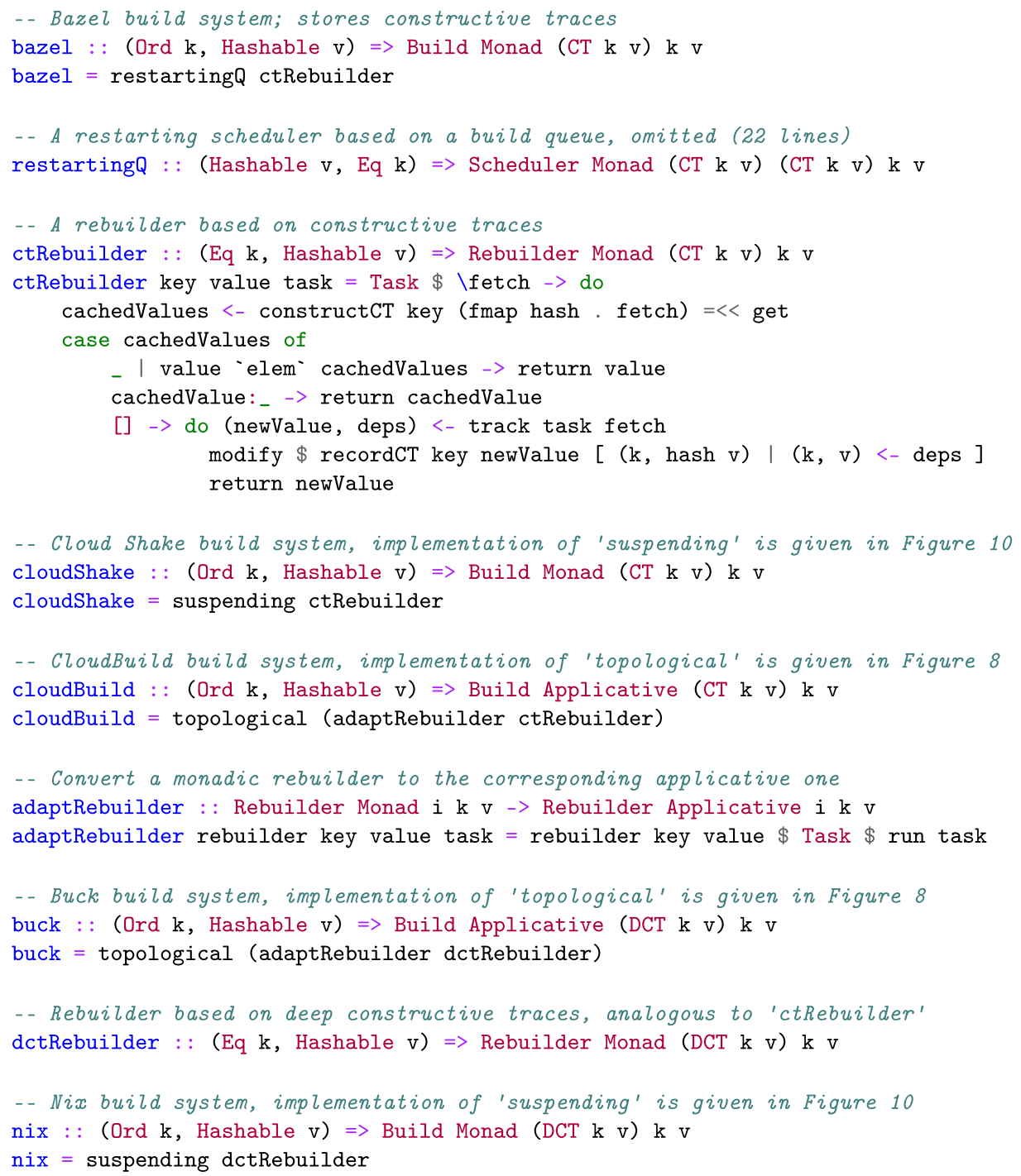

Fig. 11. Bazel, Cloud Shake, CloudBuild, BUCK and NiX in our framework.

executing the task. The obtained newValue and the task's dependencies are recorded as a new constructive trace for future use.

The BAZEL build system uses a restarting scheduler whose implementation we omit. It is similar to EXCEL's restarting scheduler defined in Figure 9, but instead of building keys in the order specified by the persistently stored calc chain, BAZEL uses a build queue. The build starts with the queue containing all dirty keys. Similarly to EXCEL, the rebuilding of a key extracted from the queue may fail because one of its dynamic dependencies is dirty. In this case, the key is marked as blocked and its rebuilding is deferred. Whenever a key is successfully rebuilt, all keys that were previously blocked on it are added back to the queue and their build is eventually restarted. 
Note that although both our model and BAZEL's actual implementation supports dynamic dependencies, it is currently not possible to define new monadic build rules in the language available to users. Instead, users have to rely on a collection of predefined built-in rules, which cover many common instances of dynamic dependencies.

By switching to the topological scheduler, we obtain a model of Microsoft's CLOUdBuILD - an applicative build system that combines conventional scheduling of statically known directed acyclic graphs with constructive traces (Esfahani et al., 2016). We convert a monadic ctRebuilder into an applicative one by applying an adapter adaptRebuilder, which unwraps a given Task Applicative and wraps it into Task Monad.

Our models of BUCK (Facebook, 2013) and Nix (Dolstra et al., 2004) use the rebuilder based on deep constructive traces (Section 5.4), called dctRebuilder, whose implementation we omit since it is very similar to that of ctRebuilder. BUCK uses the topological scheduler and is an applicative build system, whereas NIX uses the suspending scheduler and is therefore monadic.

Using the abstractions built thus far, we have shown how to compose schedulers with rebuilders to reproduce existing build systems. To us, the most interesting build system as yet unavailable would compose a suspending scheduler with constructive traces - providing a cloud-capable build system that is minimal and supports both early cutoff and monadic dependencies. Using our framework, it is possible to define and test such a system, which we call Cloud SHAKE. All we need to do is compose suspending with ctRebuilder, as shown in Figure 11.

\section{Experience}

We have presented a framework that can describe, and indeed execute in prototype form, a wide spectrum of build systems. But our ultimate goal is a practical one: to use these insights to develop better build systems. Our earlier work on SHAKE (Mitchell, 2012), and applying SHAKE to building Glasgow Haskell Compiler (GHC) (Mokhov et al., 2016), makes progress in that direction.

Based on the theory developed in this paper, we have extended SHAKE to become Cloud SHAKe, the first cloud-capable build system to support both early cutoff and monadic dependencies (Section 6.5), and used it to implement GHC's (very substantial) build system, HADRIAN (Mokhov et al., 2016). In this section, we reflect on our experience of turning theory into practice.

\subsection{Haskell as a design language}

Build systems are surprisingly tricky. It is easy to waffle and remarkably hard to be precise. As this paper exemplifies, it is possible to use Haskell as a design language, to express quite abstract ideas in a precise form - indeed, precise enough to be executed.

Moreover, doing so is extremely beneficial. The discipline of writing executable prototypes in Haskell had a profound effect on our thinking. It forced misconceptions to surface early. It required us to be explicit about side effects. It gave us a huge incentive to design abstractions that had simple types and an explicable purpose. 
Consider, for example, our Task type:

newtype Task $c k$ v $=$ Task (forall $\mathrm{f} . \mathrm{c} f \Rightarrow(\mathrm{k}->\mathrm{f} \mathrm{v})->\mathrm{f} \mathrm{v}$ )

We started off with a much more concrete type and explored multiple variants. During those iterations, this single line of code gave us a tangible, concrete basis for productive conversations, much more so than general debate about "tasks".

It is also worth noting that we needed a rather expressive language to faithfully express the abstractions that seem natural in this setting. In the case of Task, we needed a data constructor with a polymorphic field; a higher-kinded type variable $f:: *->*$; an even more abstracted kind for c : : $\left(*_{-}>*^{\prime}\right)->$ Constraint; and, of course, type classes. Our models have since been translated to Rust (Gandhi, 2018) and Kotlin (Estevez \& Shetty, 2019), and in both cases there was a loss of precision due to language-specific limitations.

When thinking about the type constructors over which $f$ might usefully range, it turned out that we could adopt the existing abstractions of Functor, Applicative, Monad, and so on. That in turn led us to a new taxonomy of build systems - see Section 3.4. In the other direction, trying to express an existing build system DuNE in our models led us to finding a new abstraction - the Selective type class (Mokhov et al., 2019), which turned out to be useful outside the build systems domain.

The effect of using a concrete design language went well beyond merely expressing our ideas: it directly influenced our thinking. For example, here are definitions for a scheduler and rebuilder, from Section 6.1:

type Scheduler c i ir k v $=$ Rebuilder c ir k v $\rightarrow$ Build c i k v

type Rebuilder $\mathrm{c}$ ir $\mathrm{k} \mathrm{v}=\mathrm{k} \rightarrow \mathrm{v}->$ Task $\mathrm{k} \mathrm{k} \mathrm{v} \rightarrow$ Task (MonadState ir) k v

These powerful and modular abstractions, which ultimately formed part of the conceptual structure of the paper, emerged fairly late in the project as we repeatedly reviewed, redesigned, and refactored our executable prototypes. It is hard to believe that we could have developed them without the support of Haskell as a design language.

\subsection{Experience from SHAKE}

The original design of SHAKE has not changed since the initial paper, but the implementation has continued to mature - there have been roughly 5,000 subsequent commits to the SHAKE project. ${ }^{8}$ These commits added concepts like resources (for handling situations when two build tasks contend on a single external resource), rewriting serialisation to be faster, documentation including a website, ${ }^{9}$ and add a lot of tests. The biggest change in that time period was an implementation change: moving from blocking threads to continuations for the suspending scheduler. But otherwise, almost all external and internal details remain the same. ${ }^{10}$ We consider the lack of change suggestive that SHAKE is based on fundamental principles - principles we can now name and describe as a consequence of this paper.

8 See https://github.com/ndmitchell/shake.

9 See https://shakebuild.com.

10 The most visible change is purely notational: switching from $*>$ to $\%>$ for defining rules, because a conflicting *> operator was added to the Haskell Prelude. 
There are two main aspects to the original SHAKE paper (Mitchell, 2012) that are described more clearly in this paper. Firstly, the rebuilder can now be described using verifying step traces Section 5.2.2, with a much clearer relationship to the unoptimised verifying traces of Section 5.2. Secondly, in the original paper, the tasks (there called "actions") were described in continuation-passing style using the following data type ${ }^{11}$ :

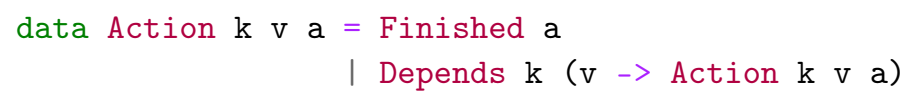

In this paper, we describe tasks more directly, in a monadic (or applicative or functorial) style. But in fact the two are equivalent: Task Monad $\mathrm{k} \mathrm{v}$ is isomorphic to Action $\mathrm{k} \mathrm{v} \mathrm{v.}$ To be concrete, the functions toAction and fromAction defined below witness the isomorphism in both directions.

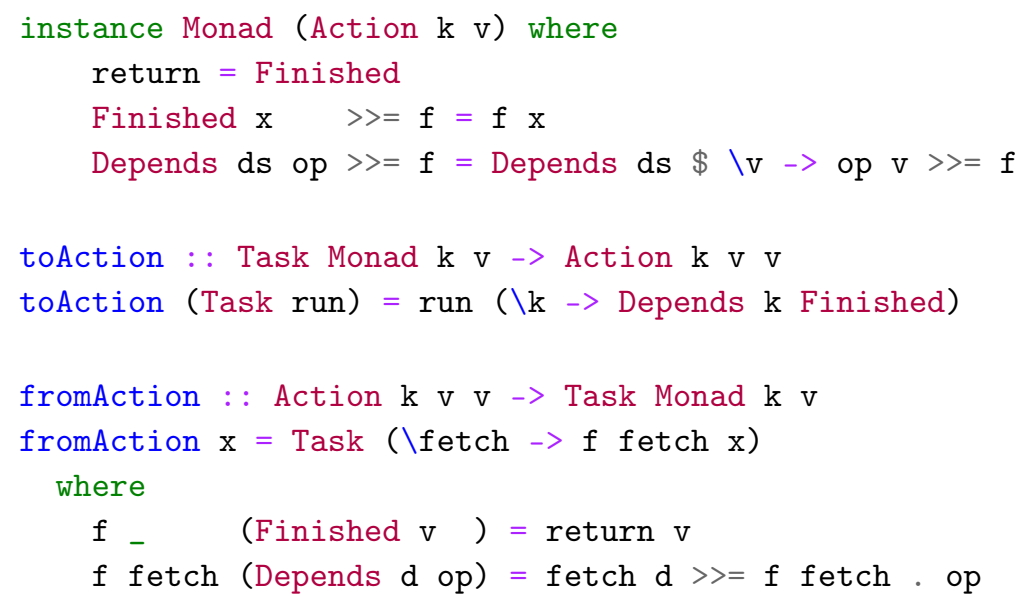

Similarly, in the original paper, MAKE tasks were described as:

data Rule $\mathrm{k} \mathrm{v} \mathrm{a}=\operatorname{Rule}\{$ depends : [k], action : [v] $->$ a $\}$

Assuming the lengths of the lists [k] and [v] always match, the data type Rule k v v is isomorphic to Task Applicative k v, and we can define a similar Applicative instance and conversion functions.

By expressing these types using Task, we are able to describe the differences more concisely (Monad versus Applicative), use existing literature to determine what is and is not possible, and explore other constraints beyond just Monad and Applicative. These and other isomorphisms for second-order functionals, that is, functions of the form

$$
\text { forall } f . c f=>(k->f \text { v) } \rightarrow \text { f a }
$$

for various choices of c are studied in depth by Jaskelioff and O'Connor (2015).

11 The original paper uses concrete types Key and Value. Here we generalise these types to $\mathrm{k}$ and $\mathrm{v}$, and also add a so that Action $\mathrm{k} \mathrm{v}$ can be an instance of Monad. 


\subsection{Experience from CLOUD SHAKE}

Converting SHAKE into CLOUd SHAKE was not a difficult process once armed with the road map in this paper. The key was the introduction of two new functions:

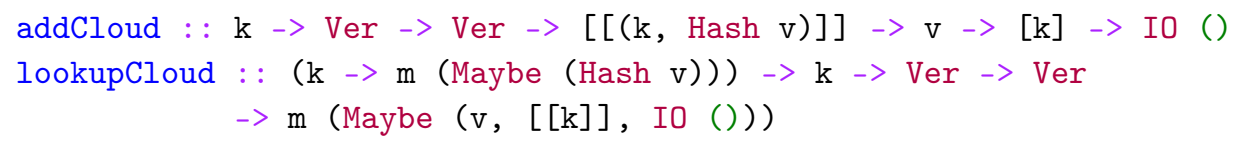

These functions are suspiciously like recordCT and constructCT from Section 5.3, with their differences perhaps the most illustrative of the changes required. ${ }^{12}$

- Two Ver arguments are passed to each function. These are the versions of the build script and the rule for this particular key. If either version changes, then it is as though the key has changed and nothing will match. These versions are important to avoid using stale build products from previous versions of the build script.

- The list of dependencies to addCloud is a list of lists, rather than a simple list. The reason is that SHAKE allows a list of dependencies to be specified simultaneously, so they can all be built in parallel.

- The addCloud function also takes a list of keys $[\mathrm{k}]$, being the files that this rule produces. These produced files include those which are output keys from a rule and those declared with the function produces.

- The lookupCloud function allows an explicit Nothing when looking up a dependent key, since some keys are not buildable.

- The lookupCloud function returns at most one result, rather than a list. This change was made for simplicity.

To integrate these functions into SHAKE, we found the most expedient route was to leave SHAKE with verifying traces, but if the verifying trace does not match, we consult the constructive trace. By bolting constructive traces onto the side of SHAKE, we avoid re-engineering of the central database. We have not found any significant downsides from the bolt-on approach thus far, so it may be a sensible route to go even if developing from scratch - allowing an optimised verified trace implementation in many cases and falling back to a more complex implementation (requiring consulting remote servers) only rarely.

The one thing we have not yet completed on the engineering side is a move to hosting caches over HTTP. At the moment all caches are on shared file systems. This approach can use mounted drives to mirror HTTP connections onto file systems, and reuse tools for managing file systems, share caches with rsync, and is simple. Unfortunately, on certain operating systems (e.g. Windows) mounting an HTTP endpoint as a file system requires administrator privileges, so an HTTP cache is still desirable.

12 We have made some minor changes from actual SHAKE, like replacing Key for $\mathrm{k}$, to reduce irrelevant differences. 


\subsection{Experience from using CLOUD SHAKE}

While we expected the GHC build system to be the first to take advantage of CLOUD SHAKE, we were actually beaten to it by Standard Chartered who reported ${ }^{13}$ :

Thanks for the symlinks release, we just finished upgrading this build system to use --share. ... Building from scratch with a warm cache takes around $5 \mathrm{~s}$, saving us up to $2 \mathrm{~h}$. Not bad!

Converting to a build suitable for sharing is not overly onerous, but nor is it trivial. In particular, a cloud build is less forgiving about untracked operations - things that are wrong but usually harmless in local builds often cause serious problems in a cloud setting. Some things that require attention in moving to a cloud build:

- Irrelevant differences: A common problem is that you do not get shared caching when you want it. As one example, imagine two users install gcc on different paths (say/usr/bin/gcc and /usr/local/bin/gcc). If these paths are recorded by the build system, the users will not share cache entries. As another example, consider a compiler that embeds the current time in the output: any users who build that file locally will not get any shared caching of subsequent outputs. Possible solutions include using relative paths; depending only on version numbers for system binaries (e.g. gcc); controlling the environment closely (e.g. using Nix); and extra flags to encourage compilers to be more deterministic.

- Insufficient produced files: A build rule must declare all files it produces, so these can be included in the cache. As an example using Haskell, compilation of Foo.hs produces Foo.hi and Foo.o. If you declare the rule as producing Foo.hi, and other rules depend on Foo.hi, but also use Foo.o after depending on Foo.hi, a local build will probably work (although treating Foo.o as a proper dependency would definitely be preferable). However, if Foo.hi is downloaded from a remote cache, Foo.o will not be present and subsequent commands may fail (e.g. linking). In practice, most issues encountered during the move to cloud builds for GHC were caused by failing to declare produced files.

- Missing dependencies: While missing dependencies are always a problem, the move to a cloud build makes them more serious. With local builds, outputs will be built at least once per user, but with a cloud build they might only be built once ever.

To help with the final two issues - insufficient dependencies and produced files - we have further enhanced the SHAKE lint modes, coupling them to a utility called FSATrace, which detects which files are read/written by a command line execution. Such information has been very helpful in making the GHC build cloud ready (Eichmann, 2019).

\subsection{Experience from building GHC with SHAKE}

HADRIAN is a build system for the Glasgow Haskell Compiler (The GHC Team, 2019). It was developed to replace a MAKE-based build system and solve multiple scalability and

13 https://groups.google.com/d/msg/shake-build-system/NbB5kMFS34I/mZ9L4TgkBwAJ 
maintainability challenges. As discussed in detail by Mokhov et al. (2016), most of these challenges were consequences of two key shortcomings of MAKE: (i) poor abstraction facilities of makefiles, notably the need to program in a single namespace of mutable string variables and (ii) the lack of dynamic dependencies (Section 2.3). HADRIAN benefits both from SHAKE's features and from the host language Haskell, making the new GHC build system easier to understand and maintain.

Interestingly, although SHAKE is not a self-tracking build system (Section 8.8), HADRIAN implements a little domain-specific language for constructing build command lines and then tracks command lines by treating them as a type of values - an example of partial self-tracking made possible by SHAKE's support for key-dependent value types (Section 8.6).

The development of CLOUd SHAKE allows GHC developers to benefit from caching build results between builds. Building GHC 8.8 from scratch takes $\sim 1$ hour on Windows using HADRIAN or the original MAKE-based build system. This time includes building the compiler itself, 29 bundled libraries, such as base (each in vanilla and profiled way), and 6 bundled executables, such as Haddock. One would hope that the build cache would be particularly useful for GHC's continuous integration system that builds and tests every commit but our experience has been mixed. In an ideal case, when a commit does not affect the resulting GHC binaries, for example, only modifies tests, HADRIAN can build GHC from scratch in just 3 minutes, by simply creating symbolic links to the previously built results stored in the cache. However, if a commit modifies the "Stage 1 GHC" executable - an intermediate compiler built as part of the GHC bootstrapping - any further cache hits become unlikely, thus limiting benefits of the build cache to Stage 1 only (Eichmann, 2019).

A small number of GHC build rules cannot be cached. These rules register libraries in the package database, and rather than producing files, they mutate a shared file. CLOUd SHAKE provides a way to manually label such build rules to exclude them from caching.

One of the benefits of using SHAKE is that we have access to high-quality build profiling information, allowing us to compute critical paths and other metrics; see Mitchell (2019) for an overview of SHAKE's profiling features. This information has shown us, for example, that more CPUs would not help (on unlimited CPUs the speed up would be less than $10 \%$ ), and that a handful of build tasks (two anomalously slow Haskell compilations and calls to slow single-threaded configure) take up a significant fraction of build time (at least 15\%).

\section{Engineering aspects}

In the previous sections, we have modelled the most critical subset of various build systems. However, like all real-world systems, there are many corners that obscure the essence. In this section, we discuss some of those details what would need to be done to capture them in our model and what the impact would be. 


\subsection{Partial stores and exceptions}

Our model assumes a world where the store is fully defined, every $\mathrm{k}$ is associated with $\mathrm{a} \mathrm{v}$, and every compute successfully completes returning a valid value. In the real world, build systems frequently deal with errors, for example, "file not found" or "compilation failed". There are many ways of modelling errors, and in this section we give three simple examples.

One simple approach is to include failures into the type of values $\mathrm{v}$, for example, to model a partial store we can use an algebraic data type isomorphic to Maybe:

\section{data Value $=$ FileNotFound $\mid$ FileContents String}

This is convenient if tasks are aware of failures. For example, a task may be able to cope with missing files, for example, if fetch "username.txt" returns FileNotFound, the task could use the literal string "User" as a default value. In this case, the task will depend on the fact that the file username.txt is missing and will need to be rebuilt if the user later creates this file. In general, we can use values of type Either e v when dealing with failures of type e. To automatically convert a "failure-free" task into an equivalent task operating with values of type Either e v, we can use the following function:

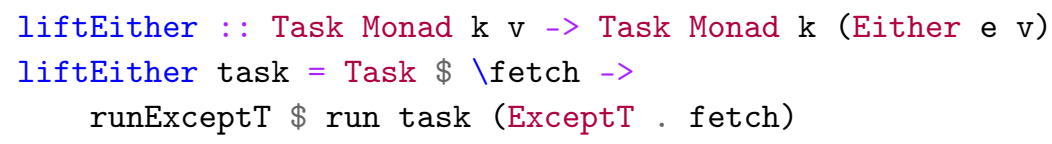

Here, liftEither wraps the result of the given fetch : : k -> Either e v into ExceptT and then runs the task in the ExceptT monad transformer (Liang et al., 1995).

Another approach is to include failures into the computation context $f$. Recall from Section 3.4 that we require tasks to be polymorphic in $f$ and can therefore choose to execute a Task in an $f$ with failures, the simplest example being $f=$ Maybe. Below we define a callback that returns Just value for keys A1 and A2 but fails with Nothing on all other keys:

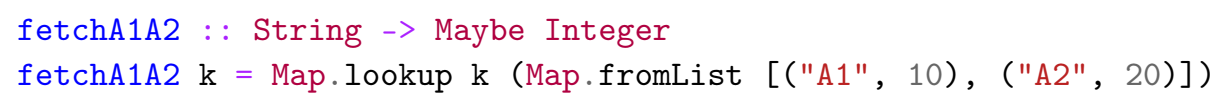

We can directly run any Task with this callback. For example, the task $B 1=A 1+A 2$ from sprsh1 in Section 3.2 returns Just 30, whereas the task B2 $=B 1 * 2$ returns Nothing because fetchA1A2 fails on B1.

This approach is convenient if tasks are not aware of failures, for example, we can model EXCEL formulae as pure arithmetic functions and introduce failures "for free" if/when needed by instantiating Tasks with an appropriate $f$. In a real system, this $f$ would be more complex than just Maybe, for example MaybeT (State Spreadsheet), thus allowing us to combine failures with access to EXCEL's spreadsheet state using the MaybeT monad transformer (Liang et al., 1995).

Finally, the task itself might not want to encode failures into the type of values $\mathrm{v}$, but instead demand that $\mathrm{f}$ has a built-in notion of failures. This can be done by choosing a 
suitable constraint c, such as Alternative, MonadPlus or even better something specific to failures, such as MonadFail. Then both the callback and the task can reuse the same failure mechanism as shown below:

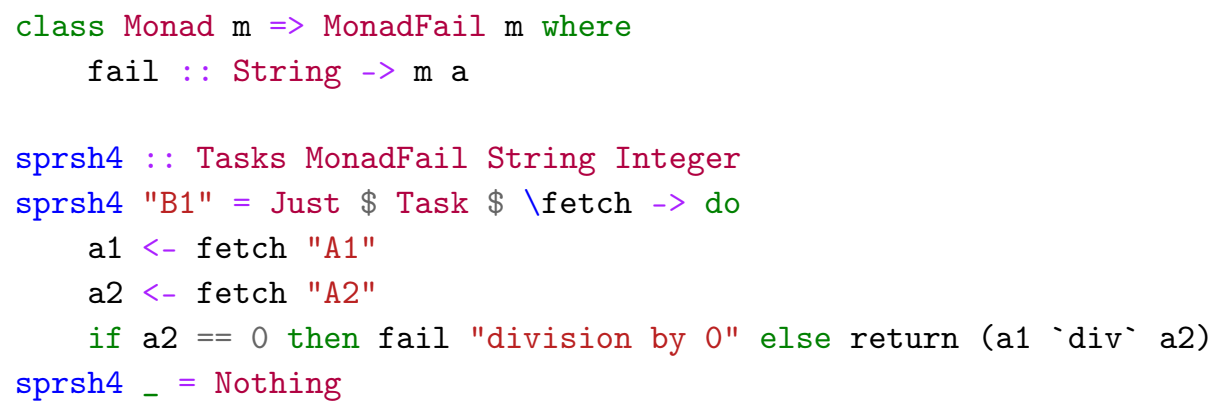

With this approach, we can implement a build system that accepts Tasks MonadFail k v and handles errors by aborting the build early and returning Either String (Store i k v) instead of just Store $i \mathrm{k} \mathrm{v}$ as in our Build abstraction Section 3.3. One possible implementation of such a failure-handling build system is based on adding an extra Either String layer into the monad stack, for example, augmenting the monad State (Store i k v) used by the schedulers in Section 6 with exceptions. We omit the actual implementation: while fairly direct, it is also tedious due to additional wrapping and unwrapping.

\subsection{Parallelism}

We have given simple implementations assuming a single thread of execution, but all the build systems we address can actually build independent keys in parallel. While it complicates the model, the complications are restricted to the scheduler:

1. The topological scheduler can build the full dependency graph, and whenever all dependencies of a task are complete, the task itself can be started.

2. The restarting scheduler can be made parallel in a few ways, but the most direct is to have $n$ threads reading keys from the build queue. As before, if a key requires a dependency not yet built, it is moved to the end - the difference is that sometimes keys will be moved to the back of the queue not because they are out of date but because of races with earlier tasks that had not yet finished. As a consequence, if the build order is persisted over successive runs (as in EXCEL), potentially racey dependencies will be separated, giving better parallelism over time.

3. The suspending scheduler can be made parallel by starting multiple dependencies in parallel. One approach is to make the request for dependencies take a list of keys, as implemented by SHAKE. Another approach is to treat the Applicative dependencies of a Task Monad in parallel, as described by Marlow et al. (2014).

Once sufficient parallelism is available, the next challenge is preventing excess parallelism and machine resource starvation, which is usually achieved with a thread pool/limit.

The actual implementation of the parallel schedulers is not overly onerous, but neither it is beautiful or informative, so we omit it. 


\subsection{Impure computations}

In our model, we define Task as a function - when given the same inputs it will always produce the same output. Alas, the real world is not so obliging. Some examples of impure tasks include:

- Untracked dependencies: Some tasks depend on untracked values - for example, C compilation will explicitly list the source.c file as a dependency, but it may not record that the version of gcc is also a dependency.

- Non-determinism: Some tasks are non-deterministic, producing any result from a possible set. As an example, GHC programs compiled using parallelism can change the order in which unique variables are obtained from the name supply, producing different but semantically identical results.

- Volatility: Some tasks are defined to change in every build, for example, EXCEL provides a "function" RANDBETWEEN producing a fresh random number in a specified range on each recalculation. Similarly, build systems like MAKE and SHAKE provide volatile phony rules. The main difference from non-deterministic tasks is that volatile tasks cannot be cached. They are readily modelled as depending on a special key RealWorld whose value is changed in every build.

There is significant interplay between all three sources of impurity. Systems like BAZEL use various sandboxing techniques to guard against missing dependencies, but none are likely to capture all dependencies right down to the CPU model and microcode version. Tasks with untracked dependencies can be marked volatile, a technique EXCEL takes with the INDIRECT function, fixing the untracked dependency at the cost of minimality.

Most of the implementations in Section 6 can deal with non-determinism, apart from BUCK, which uses the assumption of task determinism to reduce the number of cloud lookups: if all tasks are deterministic, one needs just a single cloud lookup for obtaining the value of the target key from the hash of its terminal inputs. This is highly desirable not only for shallow builds, but for non-shallow builds too, because under the assumption of task determinism all intermediate values are fully determined by the terminal inputs, and can therefore be requested in a single batch query to the server.

One way of modelling non-determinism is to demand that $\mathrm{f}$ has a built-in source of non-determinism, for example, by enriching Tasks Monad to Tasks MonadRandom that has access to an additional method getRandom : : (Integer, Integer) ->m Integer, that is, a source of random numbers in a specified range. Here is a task description corresponding to a spreadsheet with the formula B1 $=A 1+\operatorname{RANDBETWEEN}(1,2)$ :

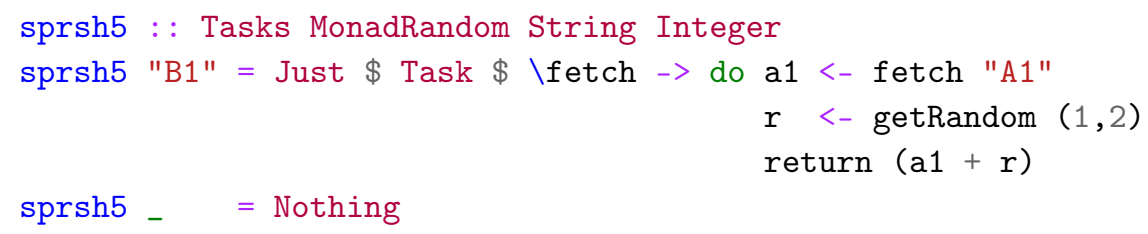

Such tasks can be modelled in our framework by adjusting the correctness definition (Section 3.6): instead of requiring that the produced value equals the result of recomputing the task, we now require that produced value belongs to the set of possible results of recomputing the task, for example, the set $\{A 1+1, A 1+2\}$ in the above example. 
Interestingly, Task MonadRandom is powerful enough to express dependency-level nondeterminism, for example, INDIRECT("A" \& RANDBETWEEN $(1,2)$ ), whereas most build tasks in real-life build systems only experience a value-level non-determinism. EXCEL handles this example simply by marking the cell volatile - an approach that can be readily adopted by any of our implementations.

\subsection{Cloud implementation}

Our model of cloud builds provides a basic framework to discuss and reason about them, but lacks a number of important engineering corners:

- Communication: When traces or contents are stored in the cloud, communication can become a bottleneck, so it is important to send only the minimum amount of information, optimising with respect to build system invariants. For example, incremental data processing systems in the cloud, such as REFLOW (GRAIL, 2017), need to efficiently orchestrate terabytes of data.

- Offloading: Once the cloud is storing build products and traces, it is possible for the cloud to also contain dedicated workers that can execute tasks remotely - offloading some of the computation and potentially running vastly more commands in parallel.

- Eviction: The cloud storage, as modelled in Section 5.3, grows indefinitely, but often resource constraints require evicting old items from the store. When evicting an old value $\mathrm{v}$, one can also evict all traces mentioning the now-defunct hash v. However, for shallow builds (see below), it is beneficial to keep these traces, allowing builds to "pass-through" hashes whose underlying values are not known, recreating them only when they must be materialised.

- Shallow builds: Building the end target, for example, an installer package, often involves many intermediate tasks. The values produced by these intermediate tasks may be large, so some cloud build systems are designed to build end targets without materialising any intermediate values, producing a so-called shallow build - see an example in Section 2.4. Some build systems go even further, integrating with the file system to only materialise the file when the user accesses it (Microsoft, 2017).

Shallow builds have a slightly weaker correctness property than in the Definition 3.6. Instead of demanding that all keys reachable from the target match, we only require matches for the target itself and the input keys reachable from the target.

As described in Section 7.4, non-determinism (Section 8.3) is harmful for cloud builds, reducing the number of cache hits. However, for deep constructive traces (Section 5.4) it is much worse, even leading to incorrect results. Figure 12 shows a Frankenbuild Esfahani et al. (2016) example, where the target report.txt, which is downloaded from the cloud, is inconsistent with its immediate dependency main.prof. This inconsistency is caused by two factors: (i) inherent non-determinism of profiling - running a profiling tool on the very same main.exe will produce different main.prof results every time; and (ii) relying on deep constructive traces that cache build results based on the hashes of terminal task inputs (in this case main.exe). The result violates all three definitions of correctness: the main definition (Section 3.6), the variant for non-deterministic tasks (Section 8.3), and the variant for shallow builds (this section). 

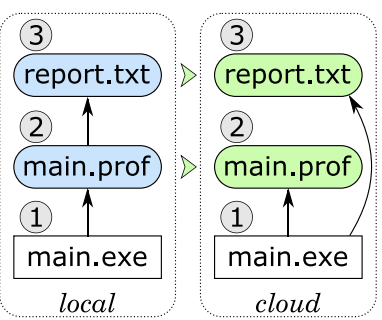
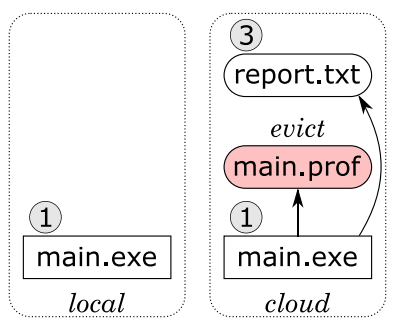

(b) Clean up, evict main.prof

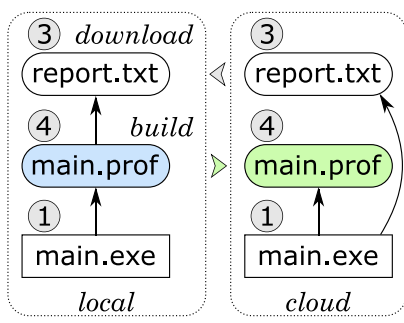

(c) Build main.prof and report.txt

Fig. 12. Frankenbuild example: (a) build a human-readable profiling report for main exe from information dump main.prof produced by a profiling tool, saving deep constructive traces in the cloud, (b) remove built files locally and evict main. prof from the cloud storage, (c) rebuild main. prof (profiling is non-deterministic, hence a new hash value), then build report.txt by downloading it from the matching deep constructive trace in the cloud. The result is a Frankenbuild because main.prof and report.txt are inconsistent. New and evicted cloud storage entries are highlighted; file hashes are shown in circles.

\subsection{Iterative computations}

Some computations are best described not by a chain of acyclic dependencies, but by a loop. For example, LATEX requires repeated rebuilding until it reaches a fixed point, which can be directly expressed in build systems, such as PLUTO (Erdweg et al., 2015). Another example is EXCEL, where a cell can depend on itself, for example, $A 1=A 1+1$. In such cases, EXCEL will normally not execute anything, but if the "Iterative Calculations" feature is enabled EXCEL will execute the formula for a specified maximum number $N$ of times per calculation (where $N$ is a setting that defaults to 100).

For example like LATEX, we consider the proper encoding to not be circular tasks, but a series of iterative steps, as described by Mitchell (2013). It is important that the number of executions is bounded, otherwise the build system may not terminate (a legitimate concern with LATEX, which can be put into a situation where it is bistable or diverging over multiple executions). The examples in EXCEL tend to encode either mutable state or recurrence relations. The former is only required because EXCEL inherently lacks the ability to write mutable state and the latter is probably better solved using explicit recurrence formulae.

We choose not to deal with cyclic dependencies - a choice that most build systems also follow. There are computation frameworks that support dependency cycles under the assumption that tasks are monotonic in a certain sense (Pottier, 2009; Radul, 2009).

\subsection{Key-dependent value types}

Key-dependent value types allow a build system to work with multiple different types of values, where the type of any particular value is determined by the key. As an example of why this might be useful, consider a build system where keys can be files (whose contents are strings) or system executables (represented by their version number) - using a single type for both values reduces type safety. SHAKE permits such key-dependent value types, 
for example, see the oracle rule in Mitchell (2012), and users have remarked that this additional type safety provides a much easier expression of concepts (Mokhov et al., 2016).

We can encode key-dependent value types using generalised algebraic data types or GADTs (Peyton Jones et al., 2006). The idea is to replace the callback fetch : : $k->f v$ by its more polymorphic equivalent $f e t c h:: \mathrm{kv}->\mathrm{f} v$, where $\mathrm{k} v$ is a GADT representing keys tagged by the type $\mathrm{v}$ of corresponding values. The variable $\mathrm{k}$ has changed from kind $*$ (a type), to $*->*$ (a type function), permitting the key to constrain the type of the value. The idea is best explained by way of an example:

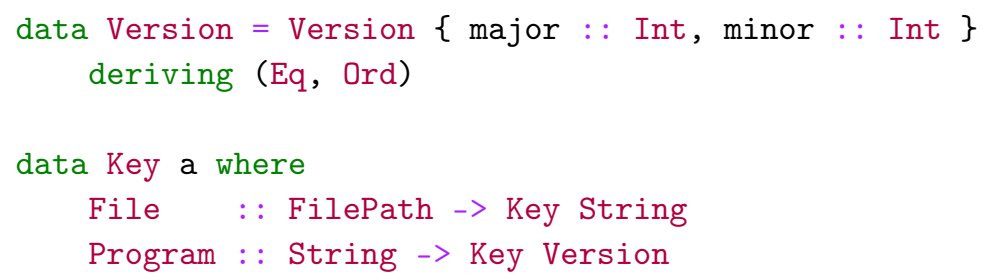

Here, we extend the usual mapping from file paths to file contents with an additional key type Program which maps the name of a program to its installed Version. The task abstraction needs to be adjusted to cope with such keys (the suffix T stands for "typed"):

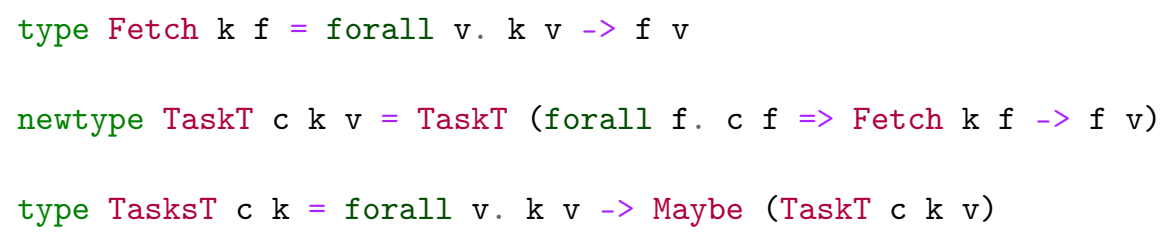

The changes compared to the definition in Section 3.2 are minimal: (i) the TaskT now uses a typed Fetch callback (we define a separate type synonym only for readability) and (ii) the type of TasksT is now polymorphic in $\mathrm{v}$ instead of being parameterised by a concrete $\mathrm{v}$. The example below demonstrates how fetch can be used to retrieve dependencies of different types: the rule release.txt concatenates the contents of two Files, while the rule main.o uses the numeric Program "gcc" to determine how the source file should be compiled.

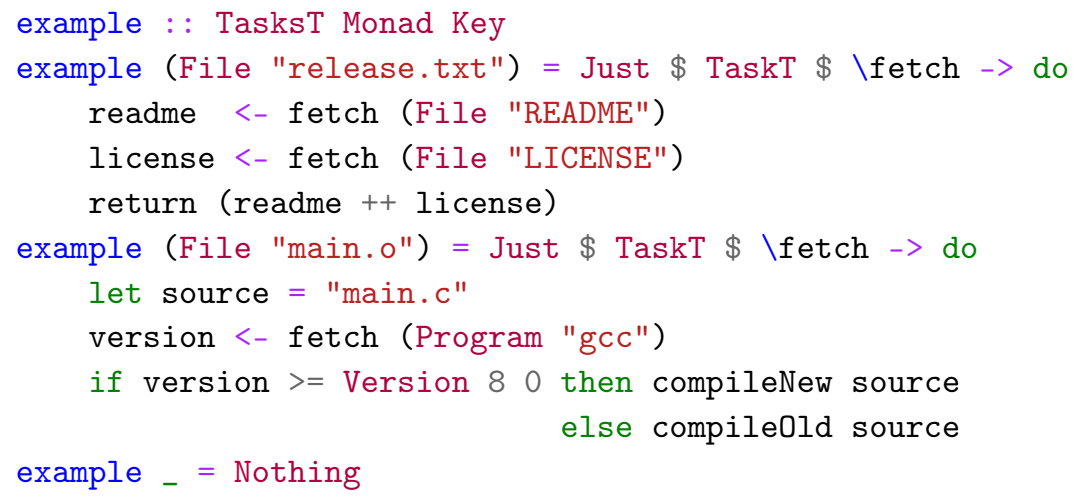


Note that like all key-dependent tasks, this example could be expressed without key dependence, at the cost of some type safety. As we will see in Sections 8.7 and 8.8, using key-dependent value types can make it easier to work with more complicated tasks.

\subsection{Multiple output build tasks}

Some build tasks produce multiple output keys, for example, ghc A.hs produces both A.hi and A.o. This pattern can be encoded by having a key A.hi+A.o which produces both results, then each of A.hi and A.o can extract the relevant result from A.hi+A.o. We can express this pattern more clearly with the extra type safety from Section 8.6:

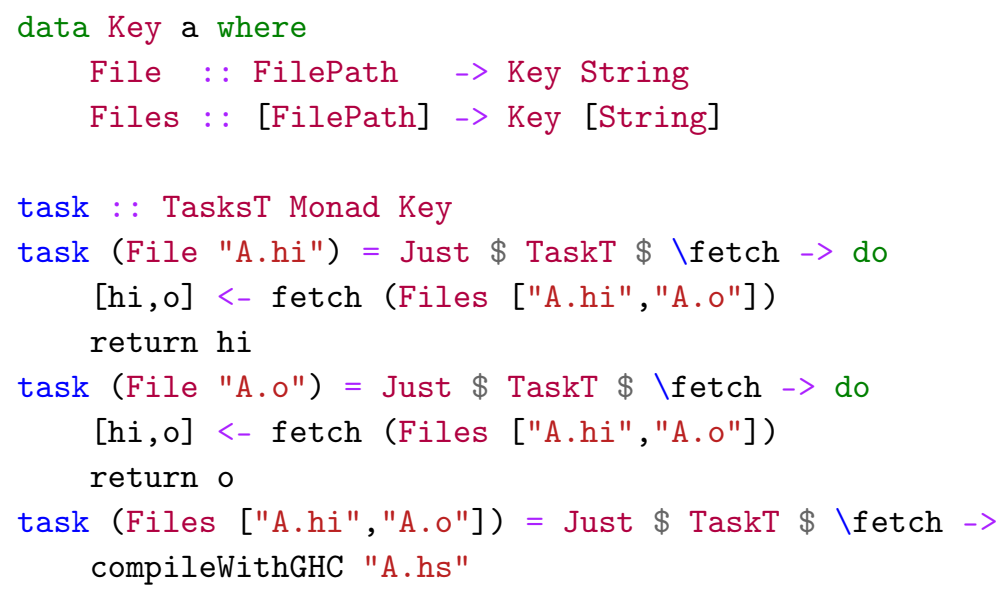

One awkward aspect is that both A.hi and A.o must ask for exactly the same Files key. If one File key swapped the order of the list to Files, then it would likely run GHC twice. To help construct a well-formed multiple output build task, it is convenient to partition the set of keys into elementary subsets. We can encode such a partition with a function from any key to all the members of its subset (in a consistent order).

type Partition $\mathrm{k}=\mathrm{k}->[\mathrm{k}]$

In our example, the partition function on either of the output names "A.hi" or "A.०" would return ["A.hi", "A.०"].

With a suitable Partition, it is possible to create a mapping that resolves File keys automatically into the correct Files key.

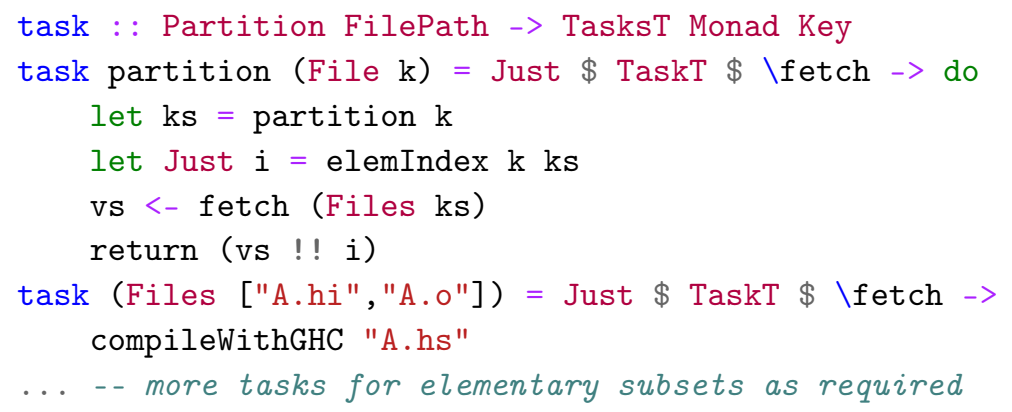


In the above function, we compute the elementary subset ks of the given key $k$, find the index of $k$ within the subset (using elemIndex), run the fetch to build every key in the subset, then extract out the value corresponding to $\mathrm{k}$ (using the indexing operation ! !).

\subsection{Self-tracking}

Some build systems, for example EXCEL and NINJA, are capable of recomputing a task if either its dependencies change, or the task itself changes. For example:

$$
\begin{aligned}
& \mathrm{A} 1=20 \\
& \mathrm{~A} 2=10
\end{aligned} \quad \mathrm{~B} 1=\mathrm{A} 1+\mathrm{A} 2
$$

In EXCEL, the user can alter the value produced by $B 1$ by either editing the inputs of $A 1$ or $A 2$, or editing the formula in $B 1$, for example, to $A 1 * A 2$. This pattern can be captured by describing the rule producing $\mathrm{B} 1$ as also depending on the value $\mathrm{B} 1$-formula. The implementation can be given very directly in a Tasks Monad - concretely, first look up the formula, then interpret it:

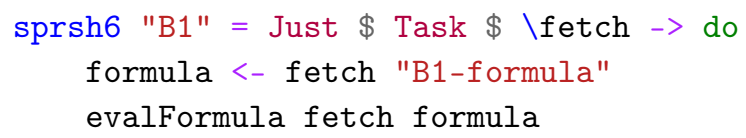

The build systems that have precise self-tracking are all ones which use a non-embedded domain-specific language to describe build tasks, that is, a task is described by a data structure (or syntax tree), and tasks can be compared for equality by comparing those data structures. Build systems that use a full programming language, for example SHAKE, are faced with the challenge of implementing equality on arbitrary tasks - and a task is just a function. For such build systems, the only safe approach is to assume (pessimistically) that any change to the build system potentially changes any build task - the classic example being build tasks depending on the makefile itself.

Below we show how to implement self-tracking in a build system that allows users to describe build tasks by scripts written in a non-embedded domain specific language. We will denote the type of scripts by $\mathrm{s}$ and will assume that scripts are indexed by keys $\mathrm{k}$ just like all other values v. More specifically, we use the following GADT to tag keys $\mathrm{k}$ with corresponding result types: $\mathrm{s}$ for scripts and $\mathrm{v}$ for other values.

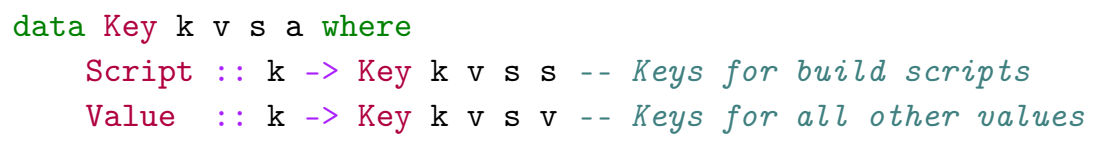

The function selfTracking defined below is a generalisation of the approach explained in the above EXCEL example sprsh6. The function takes a parser for scripts, of type s -> Task Monad k v, and a description of how to build all scripts, of type Tasks Monad k s. For sprsh6, the latter would simply fetch B1-formula when given the key B1 and return Nothing otherwise, but the presented approach can cope with much more sophisticated scenarios where scripts themselves are derived from "script sources", for example, all $\mathrm{C}$ compilation scripts can be obtained from a single pattern rule, such as 
gcc -c [source] -o [object]. The resulting typed task description TasksT Monad (Key k v s) tracks both values and scripts that compute them and is therefore self-tracking.

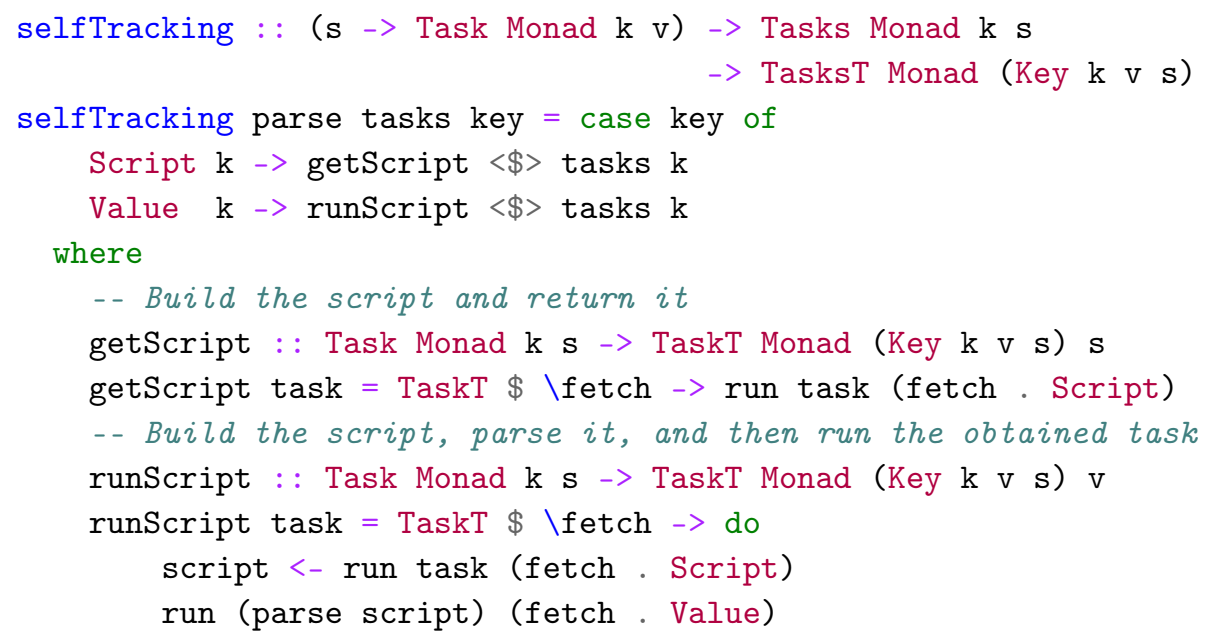

It is possible to implement selfTracking without relying on GADTs and typed tasks presented in Section 8.6, at the cost of using partial functions.

\subsection{File watching versus polling}

Most build systems use files for their inputs. When running a build, to check if a file is up to date, one option is to take the modification time or compute the content hash of each input file. However, for the largest projects, the mere act of checking the modification time for all the input files can become prohibitive. To overcome that problem, build systems targeted at large scale, for example, BAZEL and BUCK, rely on file watching API's to detect when a file has changed. Using that information, the build can avoid performing many file accesses.

File-watching build systems face new engineering challenges. Firstly, a running task may become obsolete due to a concurrent modification of its direct or transitive dependencies. To minimise the amount of unnecessary work, a scheduler can cancel the obsolete task and restart it once its dependencies are up to date. Note that topological schedulers (Section 4.1) cannot be easily adapted to support such situations, since a new topological sort may be required when file dependencies change. A further complication is that spurious dependency cycles may form during a series of file modifications, and a filewatching build system integrated with an IDE should be able to cope with such spurious cycles gracefully instead of terminating with an error.

Build systems that run continuously are also more likely to encounter errors caused by concurrent modification of build outputs. For example, if an output file is checked into the source repository, then downloading a new version of the file can interfere with the build task producing it, resulting in a corrupted output that the build system will be unable 
to detect. This problem can be solved by ensuring that tasks have an exclusive access to their outputs, for example, by sandboxing the tasks whose outputs can be modified externally.

\section{Related work}

While there is research on individual build systems, there has been little research to date comparing different build systems. In Section 2, we covered several important build systems - in this section, we relate a few other build systems to our abstractions and discuss other work where similar abstractions arise.

\subsection{Other build systems}

Most build systems, when viewed at the level we talk, can be captured with minor variations on the code presented in Section 6. Below, we list some notable examples:

- DunE (Jane Street, 2018) is a build system designed for OCaml/Reason projects. Its original implementation used arrows (Hughes, 2000) rather than monads to model dynamic dependencies, which simplified static dependency approximation. DUNE was later redesigned to use a flavour of selective functors (Mokhov et al., 2019), making it a closer fit to our abstractions.

- NinJa (Martin, 2017) combines the topological scheduler of MAKE with the verifying traces of SHAKE - our associated implementation provides such a combination. NINJA is also capable of modelling build rules that produce multiple results, a limited form of multiple value types Section 8.6.

- Nix (Dolstra et al., 2004) has coarse-grained dependencies, with precise hashing of dependencies and downloading of precomputed build products. We provided a model of NIX in Section 6.5, although it is worth noting that NIX is not primarily intended as a build system and the coarse-grained nature (packages, not individual files) makes it targeted to a different purpose.

- Pluto (Erdweg et al., 2015) is based on a similar model to SHAKE, but additionally allows cyclic build rules combined with a user-specific resolution strategy. Often such a strategy can be unfolded into the user rules without loss of precision, but a fully general resolution handler extends the Task abstraction with new features.

- Redo (Bernstein, 2003; Grosskurth, 2007; Pennarun, 2012) almost exactly matches SHAKE at the level of detail given here, differing only in aspects like rules producing multiple files Section 8.6. While REDO predates SHAKE, they were developed independently; we use SHAKE as a prototypical example of a monadic build system because its implementation presents a closer mapping to our Task abstraction.

- Tup (Shal, 2009) functions much like MAKE, but with a refined dirty-bit implementation that watches the file system for changes and can thus avoid rechecking the entire graph. TUP also automatically deletes stale results. 
The one build system we are aware of that cannot be modelled in our framework is FABRICATE by Hoyt et al. (2009). In FABRICATE, a build system is a script that is run in order, in the spirit of:

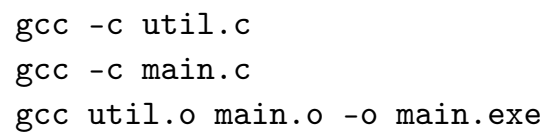

To achieve minimality, each separate command is traced at the OS level, allowing FABRICATE to record a trace entry stating that gcc -c util.c reads from util.c. In future runs, FABRICATE runs the script from start to finish, skipping any commands where no inputs have changed. The main difference from our Tasks abstraction is that instead of supplying a mapping from keys to tasks, a FABRICATE script supplies a list of build statements, in a user-scheduled order, without declaring what each statement reads or writes.

Taking our abstraction, it is possible to encode FABRICATE assuming that commands like gcc -c util.c are keys, there is a linear dependency between each successive key, and that the OS-level tracing can be lifted back as a monadic Task function. ${ }^{14}$ However, in our pure model, the mapping is not perfect as gcc writes to arbitrary files whose locations are not known in advance. One way of capturing arbitrary writes in our model is to switch from one callback fetch to two callbacks, say read and write, allowing us to track both reads and writes separately.

\subsection{Self-adjusting computation}

While not typically considered build systems, self-adjusting computation is a well-studied area, and in particular, the contrast between different formulations has been thoroughly investigated, for example, see Acar et al. (2007). Self-adjusting computations can automatically adjust to an external change to their inputs. A classic example is a self-adjusting sorting algorithm, which can efficiently (in $O(\log n)$ time where $n$ is the length of the input) recalculate the result given an incremental change of the input. While very close to build systems in spirit, self-adjusting computations are mostly used for in-memory computation and rely on the ability to dynamically allocate new keys in the store for sharing intermediate computations - an intriguing feature rarely seen in build systems (SHAKE's oracles Section 8.6 can be used to model this feature to a limited degree). Another important optimisation that self-adjusting computation engines often support is the incremental processing of deltas, where instead of marking a value as "changed to 8", one can mark it as "changed by +1 ", assuming it was equal to 7 before. When a delta is small, it can often be propagated to the output more efficiently than by recomputing the output value from scratch.

A lot of research has been dedicated to finding efficient data structures and algorithms for self-adjusting computations, with a few open-source implementations, for example, INCREMENTAL by Jane Street (2015). We plan to investigate how these insights can be utilised by build systems as future work.

\footnotetext{
14 SHAKE provides support for FABRICATE-like build systems - see Development.Shake.Forward.
} 


\subsection{Memoisation}

Memoisation is a classic optimisation technique for storing values of a function instead of recomputing them each time the function is called. Minimal build systems (Section 2.1) certainly perform memoisation: they store values instead of recomputing them each time. Memoisation can, therefore, be reduced to a minimal build system (as we demonstrate below), but not vice versa, since minimal build systems solve a more complex optimisation problem.

As a simple example of using a build system for memoisation, we solve a textbook dynamic programming problem - Levenshtein's edit distance (Levenshtein, 1966): given two input strings $a$ and $b$, find the shortest series of edit operations that transforms $a$ to $b$. The edit operations are typically inserting, deleting, or replacing a symbol. The dynamic programming solution of this problem is so widely known, for example, see Cormen et al. (2001), that we provide its encoding in our Tasks abstraction without further explanation. We address elements of strings $a_{i}$ and $b_{i}$ by keys A $i$ and $\mathrm{B} i$, respectively, while the cost of a subproblem $c_{i j}$ is identified by $\mathrm{C} i j$.

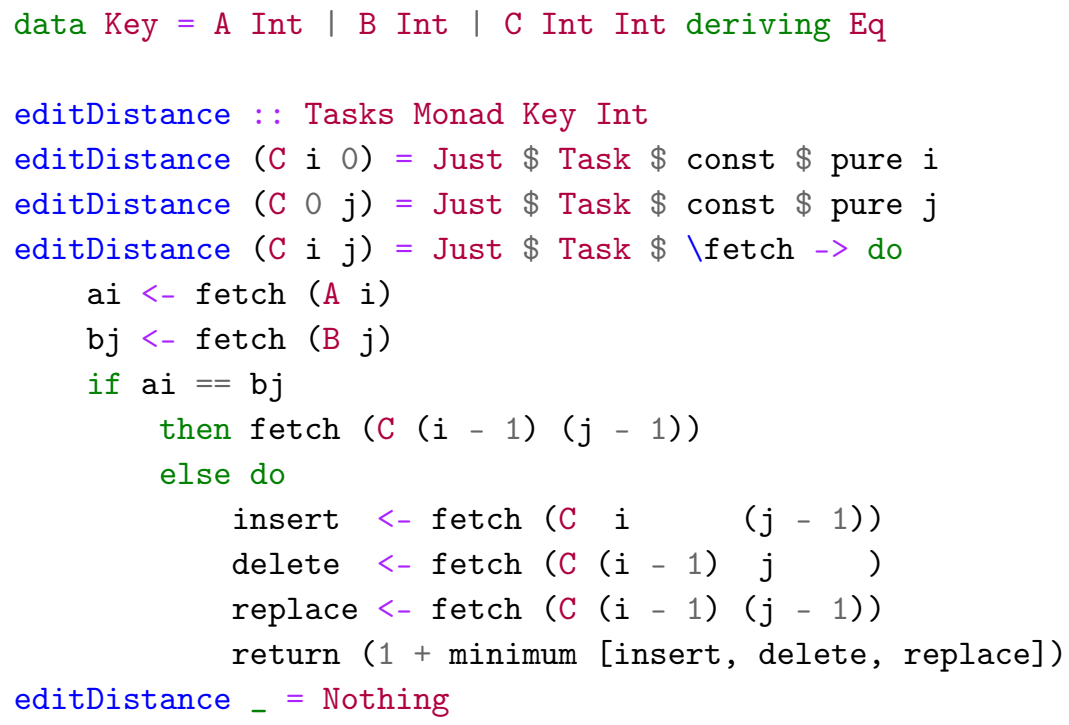

When asked to build $\mathrm{C} n \mathrm{~m}$, a minimal build system will calculate the result using memoisation. Furthermore, when an input $a_{i}$ is changed, only necessary, incremental recomputation will be performed - an optimisation that cannot be achieved just with memoisation.

Self-adjusting computation, memoisation, and build systems are inherently related topics, which poses the question of whether there is an underlying common abstraction waiting to be discovered.

\section{Conclusions}

We have investigated multiple build systems, showing how their properties are consequences of two implementation choices: what order you build in and how you decide whether to rebuild. By first decomposing the pieces, we show how to recompose the pieces 
to find new points in the design space. In particular, a simple recombination leads to a design for a monadic suspending cloud build system, which we have implemented and use in our day-to-day development.

\section{Acknowledgments}

Thanks to anonymous reviewers and everyone else who provided us with feedback on earlier drafts: Ulf Adams, Arseniy Alekseyev, Dan Bentley, Martin Brüstel, Ulan Degenbaev, Jeremie Dimino, Andrew Fitzgibbon, Georgy Lukyanov, Simon Marlow, Evan Martin, Yaron Minsky, Guillaume Maudoux, Philip Patsch, Michael Peyton Jones, Andrew Phillips, François Pottier, Rohit Ramesh, Irakli Safareli, and Zhen Zhang. Your contributions were incredibly valuable.

Andrey Mokhov's research was funded by a Royal Society Industry Fellowship IF160117 on the topic "Towards Cloud Build Systems with Dynamic Dependency Graphs".

\section{Conflicts of Interest}

None

\section{References}

Acar, U. A., Blelloch, G. E. \& Harper, R. (2002) Adaptive functional programming. In Proceedings of the 29th ACM SIGPLAN-SIGACT Symposium on Principles of Programming Languages (POPL). ACM, pp. 247-259.

Acar, U. A., Blume, M. \& Donham, J. (2007) A consistent semantics of self-adjusting computation. In European Symposium on Programming. Springer, pp. 458-474.

Bernstein, D. J. (2003) Rebuilding target files when source files have changed. Available at: http:// cr.yp.to/redo.html

Capriotti, P. \& Kaposi, A. (2014) Free Applicative Functors. vol. 153. Open Publishing Association.

Claessen, K. (1999) A poor man's concurrency monad. J. Funct. Program., 9(3), 313-323.

Cormen, T. H., Leiserson, C. E., Rivest, R. L. \& Stein, C. (2001) Introduction to Algorithms. MIT Press.

De Levie, R. (2004) Advanced Excel for Scientific Data Analysis. Oxford University Press.

Demers, A., Reps, T. \& Teitelbaum, T. (1981) Incremental evaluation for attribute grammars with application to syntax-directed editors. In Proceedings of the 8th ACM SIGPLAN-SIGACT Symposium on Principles of Programming Languages (POPL). ACM, pp. 105-116.

Dolstra, E., De Jonge, M., Visser, E., et al. (2004) Nix: A safe and policy-free system for software deployment. In LISA, vol. 4, pp. 79-92.

Eichmann, D. (2019) Exploring cloud builds in Hadrian. Available at: https://web.archive. org/web/20191008171120/https://well-typed.com/blog/2019/08/exploring-cloudbuilds-in-hadrian/

Erdweg, S., Lichter, M. \& Weiel, M. (2015) A sound and optimal incremental build system with dynamic dependencies. ACM Sigplan Notices 50(10), 89-106.

Esfahani, H., Fietz, J., Ke, Q., Kolomiets, A., Lan, E., Mavrinac, E., Schulte, W., Sanches, N. \& Kandula, S. (2016) Cloudbuild: Microsoft's distributed and caching build service. In Proceedings of the 38th International Conference on Software Engineering Companion. ACM, pp. 11-20. 
Estevez, P. \& Shetty, D. (2019) Translation of Build Systems à la Carte to Kotlin. Available at: https://web.archive.org/web/20191021224324/https://github.com/arrow-kt/ arrow/blob/paco-tsalc/modules/docs/arrow-examples/src/test/kotlin/arrow/ BuildSystemsALaCarte.kt.

Facebook. (2013) Buck: A high-performance build tool. Available at: https://buckbuild.com/

Feldman, S. I. (1979) Make - a program for maintaining computer programs. Software Pract. Exp. 9(4), 255-265.

Gandhi, V. (2018) Translation of build systems à la Carte to Rust. Available at: https://web. archive.org/web/20191020001014/https://github.com/cutculus/bsalc-alt-code/ blob/master/BSalC.rs

Google. (2016) Bazel. Available at: http://bazel.io/

GRAIL. (2017) Reflow: A system for incremental data processing in the cloud. Available at: https://github.com/grailbio/reflow

Grosskurth, A. (2007) Purely top-down software rebuilding. M.Phil. thesis, University of Waterloo.

Hoyt, B., Hoyt, B. \& Hoyt, B. (2009) Fabricate: The better build tool. Available at: https:// github.com/SimonAlfie/fabricate

Hughes, J. (2000) Generalising monads to arrows. Sci. Comput. Program. 37(1-3), 67-111.

Hykes, S. (2013) Docker container: A standardized unit of software. Available at: https://www . docker.com/what-container

Jane Street. (2015) Incremental: A library for incremental computations. Available at: https:// github.com/janestreet/incremental

Jane Street. (2018) Dune: A composable build system. Available at: https ://github. com/ocaml/ dune

Jaskelioff, M. \& O'Connor, R. (2015) A representation theorem for second-order functionals. J. Funct. Program. 25, E13.

Kosara, R. (2008) Decimal expansion of A(4,2). Available at: https://web.archive.org/web/ 20080317104411/http://www.kosara.net/thoughts/ackermann42.html

Levenshtein, V. I. (1966) Binary codes capable of correcting deletions, insertions, and reversals. In Soviet Physics Doklady, vol. 10, No 8, pp. 707-710.

Liang, S. Hudak, P. \& Jones, M. (1995) Monad transformers and modular interpreters. In Proceedings of the 22nd ACM SIGPLAN-SIGACT Symposium on Principles of Programming Languages. ACM, pp. 333-343.

Marlow, S., Brandy, L., Coens, J. \& Purdy, J. (2014) There is no fork: An abstraction for efficient, concurrent, and concise data access. ACM SIGPLAN Notices 49, 325-337.

Martin, E. (2017) Ninja build system homepage. Available at: https://ninja-build.org/

McBride, C. \& Paterson, R. (2008) Applicative programming with effects. J. Funct. Program. 18(1), $1-13$.

Microsoft. (2011) Excel recalculation (msdn documentation). Available at: https://msdn. microsoft.com/en-us/library/office/bb687891.aspx. Also available in Internet Archive https://web.archive.org/web/20180308150857/https://msdn.microsoft. com/en-us/library/office/bb687891.aspx

Microsoft. (2017) Git Virtual File System . https://www.gvfs .io/

Mitchell, N. (2012) Shake before building: Replacing Make with Haskell. ACM SIGPLAN Notices 47, 55-66.

Mitchell, N. (2013) How to write fixed point build rules in Shake. Available at: https:// stackoverflow. com/questions/14622169/how-to-write-fixed-point-build-rulesin-shake-e-g-latex

Mitchell, N. (2019) GHC rebuild times - shake profiling. Available at: https://neilmitchell. blogspot.com/2019/03/ghc-rebuild-times-shake-profiling.html

Mokhov, A., Mitchell, N., Peyton Jones, S. \& Marlow, S. (2016) Non-recursive make considered harmful: Build systems at scale. In Proceedings of the 9th International Symposium on Haskell, Haskell 2016. ACM, pp. 170-181. 
Mokhov, A., Mitchell, N. \& Peyton Jones, S. (2018) Build systems à la carte. Proc. ACM Program. Lang. 2(ICFP), 79:1-79:29.

Mokhov, A., Lukyanov, G., Marlow, S. \& Dimino, J. (2019) Selective applicative functors. Proc. ACM Program. Lang. 3(ICFP). https://dl . acm.org/doi/10.1145/3341694

Pennarun, A. (2012) Redo: A top-down software build system. Available at: https://github. com/apenwarr/redo

Peyton Jones, S., Vytiniotis, D., Weirich, S. \& Washburn, G. (2006) Simple unification-based type inference for GADTs. ACM SIGPLAN Notices 41, 50-61.

Pottier, F. (2009) Lazy least fixed points in ML. http://gallium.inria.fr/ fpottier/ publis/fpottier-fix.pdf

Radul, A. (2009) Propagation Networks: A Flexible and Expressive Substrate for Computation. Ph.D. thesis, MIT.

Shal, M. (2009) Build system rules and algorithms. Available at: http://gittup.org/tup/ build_system_rules_and_algorithms.pdf /

The GHC Team. (2019) The Glasgow Haskell Compiler homepage. Available at: https://www . haskell.org/ghc/ 